\title{
Multi-Swarm Whale Optimization Algorithm for Data Clustering Problems using Multiple Cooperative Strategies
}

\author{
Ravi Kumar Saidala \\ Acharya Nagarjuna University, Nagarjuna Nagar, 522510, India \\ E-mail: saidalaravikumar@gmail.com \\ Nagaraju Devarakonda \\ LBR College of Engineering, Mylavaram, 521230, India \\ E-mail: dnagaraj_dnr@yahoo.co.in
}

Received: 28 July 2017; Accepted: 11 September 2017; Published: 08 August 2018

\begin{abstract}
Computational Intelligence (CI) is an as of emerging area in addressing complex real world problems. The WOA has taken its root from the collective intelligent foraging behavior of humpback whales (Megaptera Novaeangliae). The standard WOA is suffers from the selection of best agent while whales searching and encircling prey. This research paper deals with the multi-swarm cooperative strategies for finding the best agents which balances the two phase's exploration and exploitation. The performance of invoked Multi-Swarm cooperative strategies into standard WOA i.e, MsWOA is first benchmarked on a set of 23 standard mathematical benchmark function problems which includes 7 UniModal, 6 Multi-modal and 10 fixed dimension multimodal functions. The obtained graphical and statistical results have been portrayed along with the previously established techniques. The obtained results depicts that the proposed cooperative strategies for WOA outperforms in solving optimization problems of standard benchmark functions. This paper also studies the numerical efficiency of proposed techniques on the problem of data clustering where 10 real data clustering problems have been taken from data repository https://archive.ics.uci.edu.data. Statistical results for the obtained cluster centroids, intra-cluster distances and inter-cluster distances confirms that the cooperative strategies for best whale agent selection improves the performance WOA for function optimization problems as well as data clustering problems.
\end{abstract}

Index Terms-Nature Inspired Algorithms, Metaheuristic optimization techniques, WOA, Cooperative Strategies, Data Cluster Problems.

\section{INTRODUCTION}

Computational Intelligence is the fast growing area in tending to this real world and complex problems. The majority of the computational Intelligence algorithms are inspired by nature [1]. Researchers now conceded the way that the most ideal approach to discover answers for this real world and complex problems by studying the nature, this unveiling the micro secrets in nature to produce new optimization algorithms. Population based meta-heuristic algorithms are ending up noticeably more well known in solving complex real world problems with use of no single gradient function. Modern problems are for the most part with various complex variables that are should have been utilized as a part of finding optimal solutions. The last two decades have witnessed many real world complex problems are addressed by the field of meta-heuristics. The main objective of these algorithms is enabling the optimization process in giving the best possible solution in any situation where there are an extensive number of possible solutions.

In Data Mining, cluster analysis identifies the patterns which are recognized from each other through their features, by finding the partitions that have similar characteristics. Partitioning is a collection of objects into clusters all together that items in one cluster ought to have a minimization of intra-cluster similarity and the maximization of inter cluster likeness. Finding optimal clusters or optimal structures of data is a difficult task when it comes to complex data. Many classical algorithms have been developed for discovering clusters of data however finding optimal structures of data is as yet a big challenging task. There are two main problems in clustering. The first is choice of initial number of centroids and the second one is distance function optimization problem. Numerous data researchers have demonstrated that k-Means clustering technique is the absolute best answer for discovering hidden patterns by clustering the entire dataset. Clustering also called unsupervised learning in some traditional fields, for example, machine learning.

Authors [2-6] used nature inspired algorithms to find the optimized structures by applying them to cluster techniques. For instance, in $k$-Means clustering similarity function should be minimizing centroid distance to obtain optimized clusters. i.e. minimization of sum of squared Euclidean distance of objects from respective cluster 
means shown in Eq. (1).

$$
d_{\text {min }}=\sum_{j=1}^{K} \sum_{Z_{i} \in C_{i}}\left\|Z_{i}-\mu_{j}\right\|^{2}
$$

where $\mu_{j}$ is the mean of $C_{j}$.

Let's consider an optimization problem. We have given an undertaking to distinguish the natural product sort. Each organic product has recognized three particular components they are contour, color, size. $\mathrm{X}$ is a dataset which has the above three components. Initial number of clusters and cluster centroids are chosen. I now need to cluster up the natural products into clusters in which it definitely has a place with. The clustering component is said to be good only if clustering method acquires two conditions, one is intra-cluster minimization and the other is inter-cluster maximization. Numerous classical clustering algorithms have the optimization problem, failed to deal with non-linear dataset and insensitive to the noisy or anomaly data. We assumed that, we have an optimization function that measures how "good" clustering. By clustering up the organic products, and after that calling an optimization function on each group to measure its goodness, and afterward summing up the goodness of each group, at that point we can quantify how "good" a certain set of groups is. At the point when there is an increase in the aggregate number of natural product sort and components, at that point the problem has all the earmarks of being a NP-Complete, so it can be solved with agreeing to a parallel meta-heuristic algorithm. Nature inspired meta-heuristic algorithm can optimize the non-linear functions in an efficient manner.

Table 1.a. List of Uni and Multi modal benchmark functions $\left(F_{1}\right.$ to $\left.F_{13}\right)$. where $V \_$no $=30, f_{\min }=0$

\begin{tabular}{|c|c|c|}
\hline F_Name & Objective Function & Range \\
\hline Sphere function & $\mathrm{F}_{1}(\mathrm{x})=\sum_{i=1}^{n} x_{i}^{2}$ & {$[-100,100]$} \\
\hline Schwefel 2.22 function & $\mathrm{F}_{2}(\mathrm{x})=\sum_{i=1}^{n}\left|x_{i}\right|+\prod_{i=1}^{n}\left|x_{i}\right|$ & {$[-10,10]$} \\
\hline Schwefel 1.2 function & $\mathrm{F}_{3}(\mathrm{x})=\sum_{i=1}^{n}\left(\sum_{j=1}^{i} x_{j}\right)^{2}$ & {$[-100,100]$} \\
\hline $\begin{array}{l}\text { Rotated Schwefel } 2.21 \\
\text { function }\end{array}$ & $\mathrm{F}_{4}(\mathrm{x})=\max _{\mathrm{i}}\left\{\left|x_{i}\right|, 1 \leq i \leq n\right\}$ & {$[-100,100]$} \\
\hline \multirow[t]{2}{*}{ Rosenbrock's function } & $\mathrm{F}_{5}(\mathrm{x})=\sum_{i=1}^{n-1}\left[100\left(x_{i+1}-x_{i}^{2}\right)^{2}+\left(x_{i}-1\right)^{2}\right]$ & {$[-30,30]$} \\
\hline & $\mathrm{F}_{6}(\mathrm{x})=\sum_{i=1}^{n}\left(\left[x_{i}-0.5\right]\right)^{2}$ & {$[-100,100]$} \\
\hline Quartic function & $\mathrm{F}_{7}(\mathrm{x})=\sum_{i=1}^{n} i x_{i}^{4}+$ random $[0,1)$ & {$[-1.28,1.28]$} \\
\hline Schwefel function & $\mathrm{F}_{8}(\mathrm{x})=\sum_{i=1}^{n}-x_{i}^{2} \sin \left(\sqrt{\left|x_{i}\right|}\right)\left(\right.$ Note: $\left.\mathrm{f}_{\min }=-418.9829 * 5\right)$ & {$[-500,500]$} \\
\hline Rotated Rastrigin's function & $\mathrm{F}_{9}(\mathrm{x})=\sum_{i=1}^{n}\left[x_{i}^{2}-10 \cos \left(2 \pi x_{i}\right)+10\right]$ & {$[-5.12,5.12]$} \\
\hline Ackley function & $\mathrm{F}_{10}(\mathrm{x})=-20 e^{\left(-0.2 \sqrt{\frac{1}{n}} \sum_{i=1}^{n} x_{i}^{2}\right)}-e^{\left(\frac{1}{n} \sum_{i=1}^{n} \cos \left(2 \pi x_{i}\right)\right)}+20+e$ & {$[-32,32]$} \\
\hline Griewank function & $\mathrm{F}_{11}(\mathrm{x})=\frac{1}{4000} \sum_{i=1}^{n} x_{i}^{2}-\prod_{i=1}^{n} \cos \left(\frac{x_{i}}{\sqrt{i}}\right)+1$ & {$[-600,600]$} \\
\hline \multirow[t]{2}{*}{ Penalized 1 function } & $\begin{array}{l}\mathrm{F}_{12}(\mathrm{x})=\frac{\pi}{n}\left\{10 \sin \left(\pi y_{1}\right)+\sum_{i=1}^{n-1}\left(y_{i}-1\right)^{2}\left[1+10 \sin \pi y_{i+1}{ }^{2}\right]+\left(y_{n}-1\right)^{2}\right\}+ \\
\sum_{i-1}^{n} u\left(x_{i}, 10,100,4\right)\end{array}$ & {$[-50,50]$} \\
\hline & $y_{i}=1+\frac{x_{i}+1}{4} u\left(x_{i}, a, k, m\right)=\left\{\begin{array}{lr}k\left(x_{i}-a\right)^{m} & x_{i}>a \\
0 & -a<x_{i}<a \\
k\left(-x_{i}-a\right)^{m} & x_{i}<-a\end{array}\right.$ & \\
\hline \multirow[t]{2}{*}{ Penalized 2 function } & $\mathrm{F}_{13}(\mathrm{x})$ & {$[-50,50]$} \\
\hline & $\begin{array}{l}=0.1\left\{\sin \left(3 \pi x_{1}\right)^{2}+\right. \\
\qquad \begin{array}{c}\left.\left.\sum_{i=1}^{n}\left(x_{i}-1\right)^{2}\left[1+\sin \left(3 \pi x_{i}+1\right)^{2}\right]+\left(x_{n}-1\right)^{2}\left[1+\sin 2 \pi x_{n}\right)^{2}\right]\right\} \\
\quad+\sum_{i-1}^{n} u\left(x_{i}, 5,100,4\right)\end{array}\end{array}$ & \\
\hline
\end{tabular}

Problem statement the problem of optimized structures of a dataset for clustering is formalized as takes after. Let consider a dataset $D_{T}$, where $T$ denotes the total number of data instances and $N$ be the dataset dimensions (variables) that are considered. Let the input $\mathrm{N}$ dimensional dataset. Consider a dataset $D$ featured by $P$ attributes:

$$
\mathrm{D}_{\mathrm{T}}=\left[\left\{\mathrm{x}_{1 \mathrm{~T}}, \mathrm{x}_{2 \mathrm{~T}}, \ldots, \mathrm{x}_{\mathrm{PT}}\right\}\right]^{T}
$$

Assume dataset $D_{T}$ with $N$ dimensions is partitioned into $C_{i}$ clusters, where $i=1$ to $k$, so that for each $D_{T}$ Similarity measured by distance $d$ then,

$$
\begin{gathered}
d\left(D_{T} \mid C_{i}\left(\left\{x_{1 T}, x_{2 T}, \ldots \ldots \ldots, x_{P T}\right)\right)=\right. \\
\max \left\{d\left(D_{T} \mid C_{l}\left(x_{1 T}, x_{2 T}, \ldots \ldots \ldots, x_{P T}\right)\right)\right\}
\end{gathered}
$$

$d$ is the distance between $D_{T}$ and $C_{i}$. $C_{i}$ Should meet the following conditions: 


$$
\begin{aligned}
C_{i} \neq \varnothing, C_{i} \cap C_{s}= & \varnothing \text { and } \bigcup_{i=1}^{k} C_{i}=D_{T} \text { where } i, s \\
& =1 \ldots k
\end{aligned}
$$

We utilize the term compactness to quality of a given cluster in light of intra and inter cluster minimization, maximization similarities respectively. The clustering problem minimizing in a simple manner is portrayed as takes after.

$$
d\left(D_{T}, Z\right)=\sum_{i=1}^{N} \cdot \sum_{j=1}^{k}\left\|D_{T i j}-Z_{j}\right\|^{2}
$$

where $k$ denotes the number of clusters, $N$ the number of dimensions, $D_{T i j}$ is the location of $i^{\text {th }}$ dimension of cluster $j$.

$$
\frac{1}{N_{j}} \sum_{i=1}^{N} D_{T i j}
$$

\begin{tabular}{|c|c|c|c|c|}
\hline F_Name & Objective Function & Range & V_no & $f_{\min }$ \\
\hline De jong function 5 & $\mathrm{~F}_{14}(\mathrm{x})=\left(\frac{1}{500} \sum_{j=1}^{25} \frac{1}{j+\sum_{i=1}^{2}\left(x_{i}-a_{i j}\right)^{6}}\right)^{-1}$ & {$[-65,65]$} & 2 & 1 \\
\hline Kowalik function & $\mathrm{F}_{15}(\mathrm{x})=\sum_{i=1}^{11}\left[a_{i}-\frac{x_{1}\left(b_{i}{ }^{2}+b_{i} x_{2}\right)}{b_{i}{ }^{2}+b_{i} x_{3}+x_{4}}\right]^{2}$ & {$[-5,5]$} & 4 & 0.00030 \\
\hline $\begin{array}{l}\text { Six-Hump Camel } \\
\text { Back function }\end{array}$ & $\mathrm{F}_{16}(\mathrm{x})=4 x_{1}{ }^{2}-2.1 x_{1}{ }^{4}+\frac{1}{3} x_{1}^{6}+x_{1} x_{2}-4 x_{2}{ }^{2}+4 x_{2}{ }^{4}$ & {$[-5,5]$} & 2 & 1.0316 \\
\hline $\begin{array}{l}\text { Branin RCOS } \\
\text { function }\end{array}$ & $\mathrm{F}_{17}(\mathrm{x})=\left(x_{2}-\frac{5.1}{4 \pi^{2}} x_{1}^{2}+\frac{5}{\pi} x_{1}-6\right)^{2}+10\left(1-\frac{1}{8 \pi}\right) \cos x_{1}+10$ & {$[-5,5]$} & 2 & 0.398 \\
\hline $\begin{array}{l}\text { Goldstein-Price } \\
\text { function }\end{array}$ & $\begin{aligned} & \mathrm{F}_{18}(\mathrm{x})= {\left[\begin{array}{c}\left.1+\left(x_{1}+x_{2}+1\right)^{2}\left(\begin{array}{c}19-14 x_{1}+3 x_{1}{ }^{2}-14 x_{2}+ \\
6 x_{1} x_{2}\end{array}\right)+\right] \\
3 x_{2}{ }^{2}\end{array}\right] * } \\
& {\left[30+\left(2 x_{1}-3 x_{2}\right)^{2} *\left(18-32 x_{1}+12 x_{1}{ }^{2}+\right.\right.} \\
&\left.\left.48 x_{2}-36 x_{1} x_{2}+27 x_{2}{ }^{2}\right)\right]\end{aligned}$ & {$[-2,2]$} & 2 & 3 \\
\hline Hartmann function 3 & $\mathrm{~F}_{19}(\mathrm{x})=-\sum_{i=1}^{4} c_{i} e^{\left(-\sum_{j=1}^{3} a_{i j}\left(x_{j}-p_{i j}\right)^{2}\right)}$ & {$[1,3]$} & 3 & -3.86 \\
\hline Hartmann function 6 & $\mathrm{~F}_{20}(\mathrm{x})=-\sum_{i=1}^{4} c_{i} e^{\left(-\sum_{j=1}^{6} a_{i j}\left(x_{j}-p_{i j}\right)^{2}\right)}$ & {$[0,1]$} & 6 & -3.32 \\
\hline Shekel function 5 & $\mathrm{~F}_{21}(\mathrm{x})=-\sum_{i=1}^{5}\left[\left(X-a_{i}\right)\left(X-a_{i}\right)^{T}+c_{i}\right]^{-1}$ & {$[0,10]$} & 4 & -10.1532 \\
\hline Shekel function 7 & $\mathrm{~F}_{22}(\mathrm{x})=-\sum_{i=1}^{7}\left[\left(X-a_{i}\right)\left(X-a_{i}\right)^{T}+c_{i}\right]^{-1}$ & {$[0,10]$} & 4 & -10.4028 \\
\hline Shekel function 10 & $\mathrm{~F}_{23}(\mathrm{x})=-\sum_{i=1}^{10}\left[\left(X-a_{i}\right)\left(X-a_{i}\right)^{T}+c_{i}\right]^{-1}$ & {$[0,10]$} & 4 & -10.5363 \\
\hline
\end{tabular}

$N_{i}$ is the number of dimensions in the $j^{\text {th }}$ cluster.

Table 1.b. List of fixed-dimension multimodal benchmark functions $\left(F_{14}\right.$ to $\left.F_{23}\right)$.

\section{RELATED WORK}

The most recent two decades have seen an exceptional change in the area of computational intelligence for growing popular and efficient optimization techniques which are generally inspired by the nature; these are well known in tackling complex np-hard problems by exploring and mimicking different phenomena of the nature. The absolute most well-known optimization algorithms are comprehensively listed below.

Darwin's theory of evolution has inspired towards the development of GA (Genetic Algorithms) [7]. These algorithms are adaptive heuristic search algorithms intended to imitate the processes in natural system. The basic idea behind the development of GA is the evolution of new chromosomes from the combinations of initial chromosome populations making an expectation that recently evolved ones are better to the old set of chromosomes. The searching capability of GA is exploited, keeping in mind the end goal to search for proper cluster centers in the feature space to such an extent that a similarity metric of the resulting cluster is optimized.

The chromosomes that which are represented as strings of real numbers, encode the centers of a fixed number of clusters [8]. The ACO [9] is probabilistic based method and was planned in view of the natural phenomenon of real ant colonies. It is a populace based MHO calculation motivated by the ant behavior that is used to determine discrete- optimization problems. Many researchers [10], [11] used ACO as tool for finding $k$ optimal clusters of $\mathrm{N}$ data objects. This algorithm has been devised and used on several artificial and real datasets. DE [12] is a population of candidate solutions based optimization technique has paved the path by storn and price and it is used to optimize real parameter, real valued functions. It is the one of the quick, robust and efficient global search heuristics of current intrigue. Many authors utilized DE for finding optimized clusters of large unlabeled datasets. As opposed to the vast majority of the current clustering techniques, the DE requires no prior knowledge of the data for finding optimal clusters [13], [14]. The behavioral aspect of many birds flocking and fish schooling patterns has prompted the development of PSO [15] by Russell Eberhart and J. Kennedy. The key idea has been created from a flock of birds where every individual in the flock determines its closest neighbor and replaces their velocity with that neighbor. Among the numerous nature-inspired algorithms, clustering using PSO technique has identified as robust and efficient in 
solving clustering problems [16]. PSO can be utilized to discover centroids of a user indicated number of clusters. It is suitable for clustering complex and linearly nonseparable datasets [17]. Keeping in mind the end goal to enhance the efficiency of PSO technique, many researchers have proposed distinctive variations of the PSO algorithm furthermore, have grown new thoughts, for example, adaptive heuristics, different fitness functions, kernel-induced similarity measures, and evolution of swarm generations and so on [18-24]. Newton's law of gravity has given rise to an optimization algorithm named as GSA [25]. It is a stochastic population based $\mathrm{MHO}$ algorithm was developed based on the gravity and mass interactions. Variants of GSA also developed based on the concept of antigravity. In GSA randomly created candidate solutions for data clustering problem then the interaction have been made to each and other via Newton's gravitation law to search problem space [26], [27]. The WOA (Whale Optimization Algorithm) [28] has been devised based on the foraging strategy of hump back whales. The strategy of searching for prey, encircling strategy and the mass net searching towards getting the prey was considered in building up this algorithm. The performance comparisons have been made by testing on 23 standard mathematical benchmark functions. Found that WOA perform efficient than many state-of-the-art optimization algorithms including PSO [28]. Authors in [29] improved the optimization performance by introducing new convergence factor in both exploration and exploitation phase. Authors [30] proposed a new parallel metaheuristic optimization algorithm (NPMOA) which was formulated by hybridizing WOA with CSA. The above variants of WOA (IWAO, CSAWOA) were tested and applied to data clustering problems [31], [32].

In this paper we use the multi-swarm cooperative strategies for enhancing the performance of standard WOA and applying it to data clustering problems. The paper focuses on ring, master-slave and hybrid cooperative strategies for finding the best agent vector $\vec{X}^{*}$. As earlier said this paper comprises two parts where in the first part we use each multi-swarm cooperative strategy and find the performance. In second part applying the swarm cooperative strategies based WOA for data clustering problems.

The structural organization of this paper is done as follows. The section III presents the Standard Whale Optimization Algorithm. In the Section IV, we have described the swarm cooperative strategies where in A, B and $\mathrm{C}$ we presented Ring, Master-Slave and Hybrid cooperative strategies respectively. The performance comparisons of swarm cooperative strategies based WOA with GSA, DE, PSO, WOA and variants of WOA have been made in Section $\mathrm{V}$ whereas Section A and B outlines the performance comparisons on 23 standard benchmark functions that are listed in Table 1. a) and b) and data clustering problems that are described in Table 6 respectively. The last section concludes the whole paper and points out the future scope.

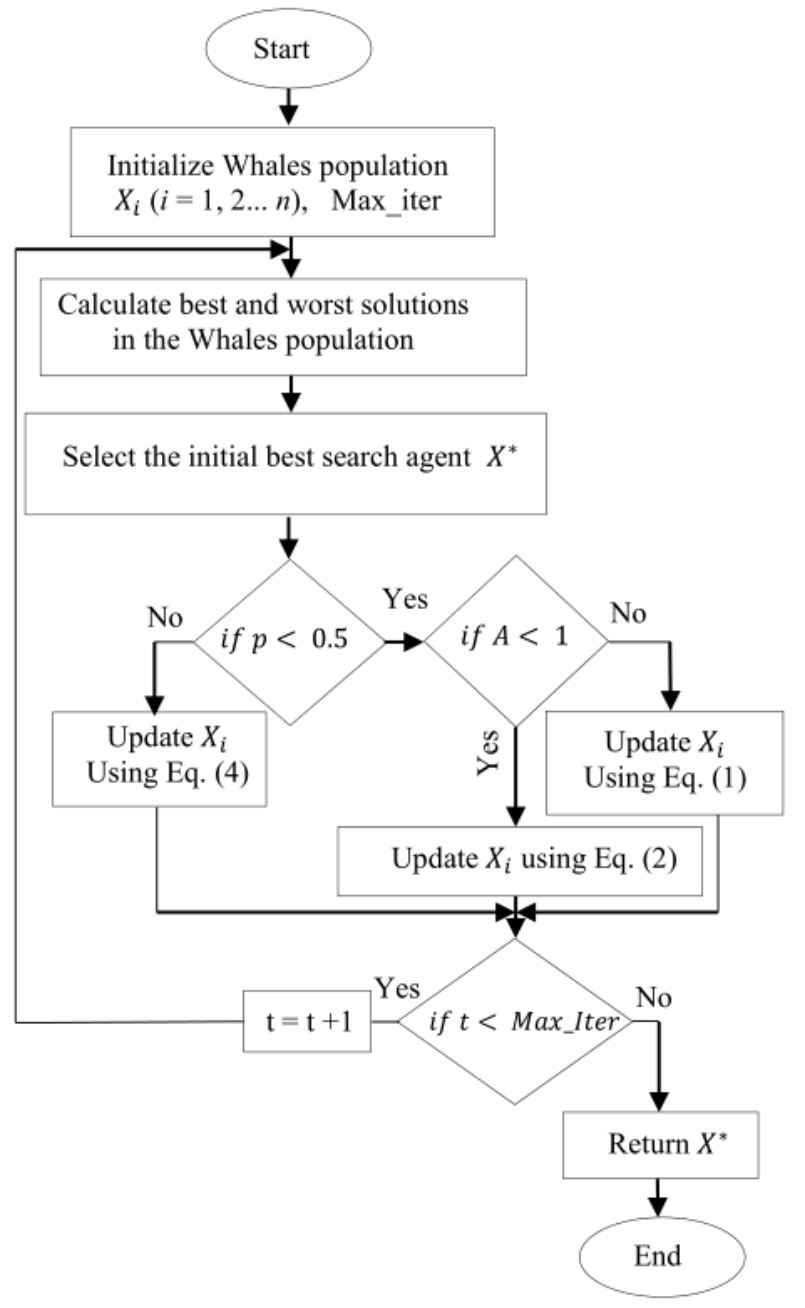

Fig.1. Flowchart of Standard Whale Optimization algorithm

\section{STANDARD WOA}

The Whale optimization Algorithm in short WAO was first coined by S. Mirjalili and A. Lewis. The special hunting behavior of humpback whales has paved the path for developing this algorithm [25]. The specialty of whales in hunting prey is they release bubbles in either 'circular' manner or ' 9 ' shaped path to move prey to the surface of the ocean. The mathematical model of this behavior is explained in two phases. The first phase is explained as hunting behavior. In this phase observed that whales show two maneuvers. They are shrinking encircling mechanism and Spiral updating position. Eq. (6) and (7) specifies the equations for the whales updating position in circular and spiral path respectively.

$$
\vec{X}_{t+1}= \begin{cases}\overrightarrow{X^{*}}{ }_{t}-\vec{A} \cdot \vec{D}, & p<0.5 \\ \overrightarrow{D^{\prime}} \cdot e^{b l} \cdot \cos (2 \pi l)+{\overrightarrow{X^{*}}}_{t} & p \geq 0.5\end{cases}
$$

where $\overrightarrow{D^{\prime}}=\left|\overrightarrow{X^{*}}{ }_{t}-X_{t}^{*}\right|, p=(-1) \times \times \operatorname{rand}(0,1)$. In Eq. (6) $\vec{a}$ is decreased to achieve to shrinking the encircling mechanism. Then $\vec{A}=(-2 \mathrm{a}) . * \operatorname{rand}(1,1)+\mathrm{a}$. where, 'a' is an integer and coefficient $\vec{A}$ is decreased by $\vec{a}$. 
In Eq. (2) constant $b$ is used in defining the shape of the logarithmic spiral, $l=(-2) \cdot * \operatorname{rand}(1,1)+1$, and '. is a dot product operator. WOA assumes probability of $50 \%$ to choose between either these two approaches. If $p<0.5$ whales updates their position in shrinking encircling mechanism else if $p \geq 0.5$, they move in Spiral updating position. The second phase is presented as searching phase. In this phase, WOA assumed that whales search for prey in two different possible ways. If $\vec{A} \geq 1$ then the updated position of whale using Eq. (8).

$$
\vec{X}_{t+1} \leftarrow \vec{X}_{\text {rand }}-\vec{A} \cdot \vec{D}
$$

where, $\vec{D}=\left|\vec{A} \cdot \vec{X}_{\text {rand }}-\vec{X}\right|$, $\vec{X}_{\text {rand }}$ is a random position vector.

Else if $\vec{A}<1$ new updated position becomes the Eq. (9).

$$
\vec{X}_{t+1} \leftarrow \vec{X}_{t}-\vec{A} \cdot \vec{D}
$$

where, $\quad \vec{D}=\left|\vec{C} \cdot \vec{X}^{*}{ }_{t}-\overrightarrow{X_{t}}\right| \vec{A}$ and $\vec{C}$ are internal parameters, the subscript ' $t$ ' specifies the current iteration, $\vec{X}$ is the position vector, $\overrightarrow{X^{*}}$ is the new position vector. '| l', ' ' are absolute value and element-by-element multiplication respectively. Both $\vec{A}$ and $\vec{C}$ are coefficient vectors where, $\vec{A}=2 \vec{a} \cdot \vec{r}-\vec{a}$, $\vec{C}=2 . \vec{r}$. Here, $\vec{a}$ straightaway decreases from 2 to 0 during the course of maneuver (in both phases: exploration and exploitation) and $\vec{r}=(-1)$.* rand $(0,1)$. The flowchart and pseudo code of WOA is given in Fig. 1 and Table 2 respectively.

\begin{tabular}{|c|c|}
\hline 1 & Initialize population $\mathbf{X}_{\mathbf{i}}(\mathbf{i}=\mathbf{1}, \mathbf{2}, \ldots, \mathbf{n})$ \\
\hline 2 & Find the fitness of each search agent \\
\hline 3 & $\mathbf{X}^{*}$ is the best search agent obtained \\
\hline 4 & $\begin{array}{l}\text { Update a, A, C, } 1 \text { and p every search } \\
\text { agent }\end{array}$ \\
\hline 5 & if1 $p<0.5$ \\
\hline 6 & if2 $|\mathbf{A}|<1$ \\
\hline 7 & Update position using Eq. (9) \\
\hline 8 & else if2 $\mathbf{A} \geq 1$ \\
\hline 9 & $\boldsymbol{X}_{\text {rand }} \quad$ Select a random search agent \\
\hline 10 & (8) Update the position using Eq. \\
\hline 11 & end if 2 \\
\hline 12 & else ifl $p \geq 0.5$ \\
\hline 13 & Update the topography using Eq. (7) \\
\hline 14 & end if 1 \\
\hline 15 & $\begin{array}{l}\text { Check is any search agent goes beyond } \\
\text { the search space and amend it }\end{array}$ \\
\hline 16 & $\begin{array}{l}\text { Calculate the fitness of each search } \\
\text { agent }\end{array}$ \\
\hline 17 & Update $\mathbf{X}^{*}$ if there is a better solution \\
\hline 18 & Go to step 4 t times and return $\mathbf{X}^{*}$ \\
\hline
\end{tabular}

Table 2. Pseudo code of Standard WOA

\section{Multi-Swarm COOPERATIVE STRATEGIES}

This section presents the multi-swarm cooperative strategies for enhancing the performance of standard WOA by invoking the Ring, Master-Slave and Hybrid strategies individually for finding the best whale population vector. The main objective of invoking these three cooperative strategies is maintaining the balancing between exploration and exploitation phases. According to the standard WOA all the whales are attracted by the best whale position. If the whales in each sub population converge at the best whale position then within a few iteration they will move near to the surface. In invoking Cooperative Strategies into Standard WOA, The whole population is grouped into multiple-cooperative swarms, we named it as sub-swarms. These sub-swarms perform different searching behavior for finding the best whale position vector Best_Sub_Pop ${ }_{k}{ }^{*}$. This phenomenon in each whale sub-population leads to the similar updating behavior and the loss of diversity in whales. The fallowing section 4.1, 4.2 and 4.3 describes the Ring, Master-Slave and Hybrid cooperative strategies with neat flowchart and pseudo code.

\section{A. Ring Cooperative Strategy}

This Strategy is a heterogeneous search strategy based on $k$-sub-swarms. The basic idea behind this strategy was inspired from ring topology. It is one of the network topology called ring network or ring topology. Many cooperative algorithms used this cooperative strategy to improve the performance of algorithm. In this cooperative strategy each sub-population of $k$ subpopulation evolves in parallel to find best whale position. Each whale sub-population interchanges its best whale position with the other whale sub-population. Each subpopulation replaces its best whale position only if gets the better whale position than the present best whale position. The interchange of best whale position happens continuously. In the optimal model structures of whale population, each sub-swarm can learn from the best whale position given by other sub-swarm and enhance their foraging direction i.e., bringing the prey closer to the surface which is optimal place to catch the prey. The pseudo code and flowchart is given in Table 3 and Fig. 2.

\section{B. Master Slave Cooperative Strategy}

This section presents the improved strategy of ring strategy. We mentioned "improved strategy of ring strategy" because the best whale position is interchanged like the ring cooperative strategy. But the difference is instead of interchanging best whale position between each other sub whale population, one sub whale population acts as master and all remaining are as slaves. it also called as multi-population collaborative strategy. In this cooperative strategy each slave sub-population of k-1 sub-population, evolves in parallel to obtain best whale position and share this information to the master. Master sub populations find the best among the received and share the same to the slaves. Master-Slave strategy balances the exploration and exploitation phases. The pseudo code and flowchart is given in Table 4 and Fig. 3. 


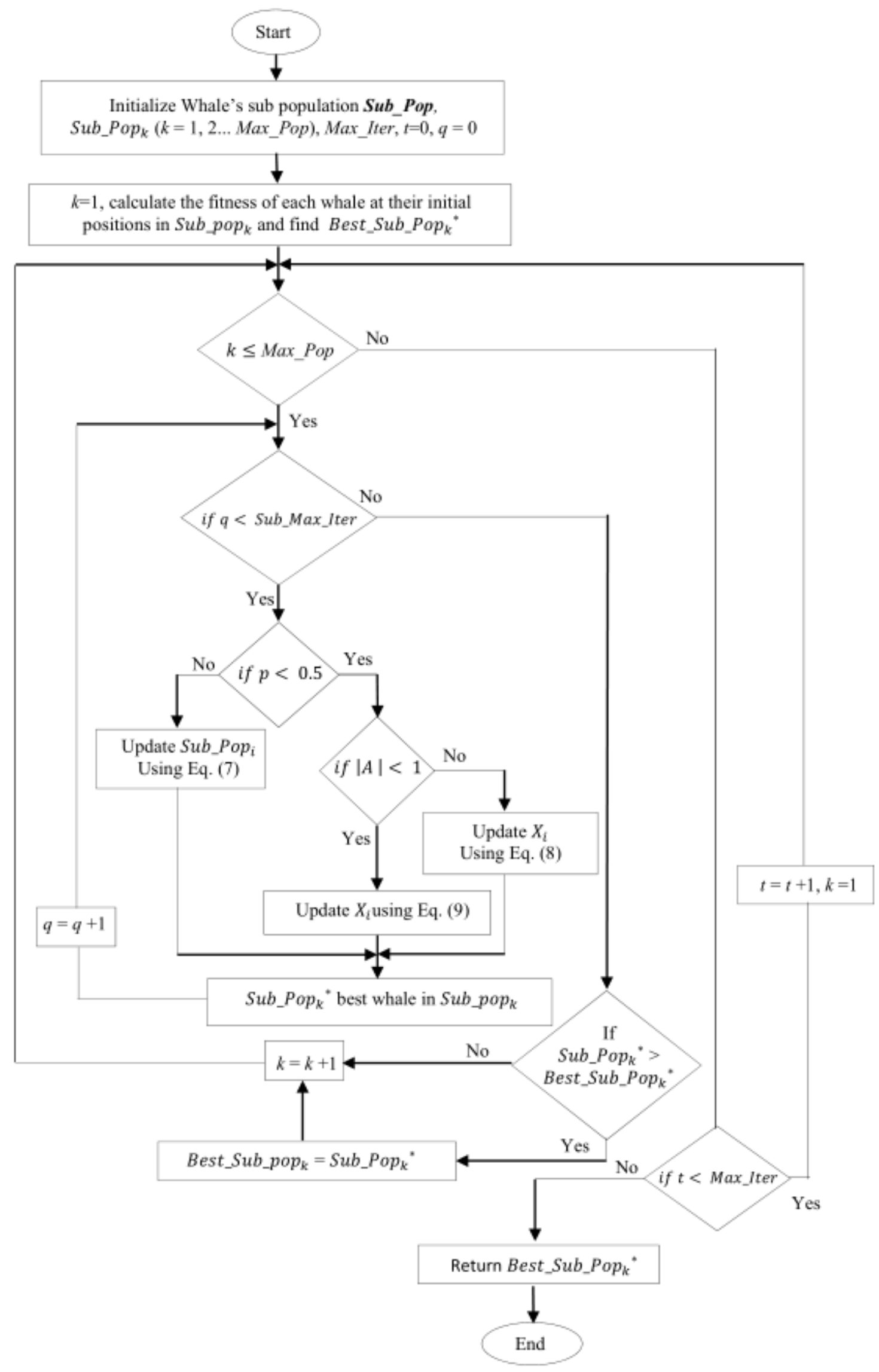

Fig.2. WOA invoking ring cooperative strategy 
Table 3. Pseudo code for WOA invoking ring cooperative strategy

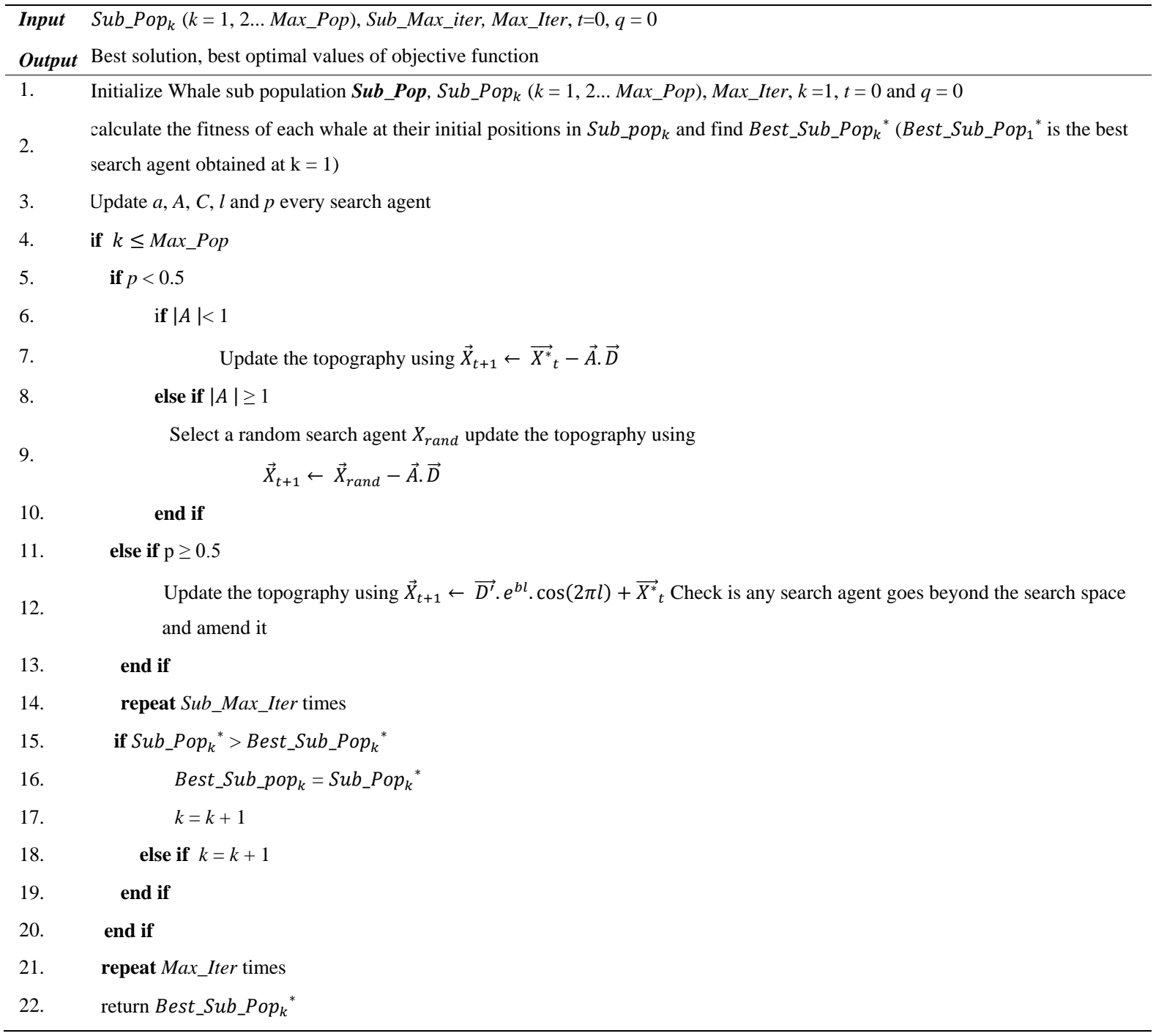

\section{Hybrid Cooperative Strategy}

This section gives the detailed description of a new hybrid cooperative strategy. The discussed cooperative strategies in earlier sections 4.1 and 4.2 are combined and form a new hybrid cooperative strategy. The key objective of this hybridization is infusion of swarm cooperative mechanism of ring cooperative strategy between the slave populations into the Master-Slave cooperative strategy. In this, a duplicate set of the best whale position of each slave sub-swarm population is shared to the master sub-swarm population. Moreover, if after Max iter (a maximum number of iterations) there is no improvement in the obtained solution, each slave subswarm population shares its best whale position obtained with its neighbors. Finally, the general best whale position is detected by the master sub-population.

The cooperation and communication model within the ring cooperative strategy are injected into the master and slave sub-populations and interchanging the individual best whale position of each slave sub-population with master sub-population reduces the possibility of moving outwards from the optimal solution. This hybridization process goes for maximizing the possibility of finding the best position within a low solution cost and the diversification of search can be achieved through the same. On the other hand, as the swarms are searching independently and in real parallelism, comprehensively the algorithm must be more effective than the previous ones. The pseudo code and flowchart of this cooperative strategy are given in Table 5 and Fig. 4 respectively. 


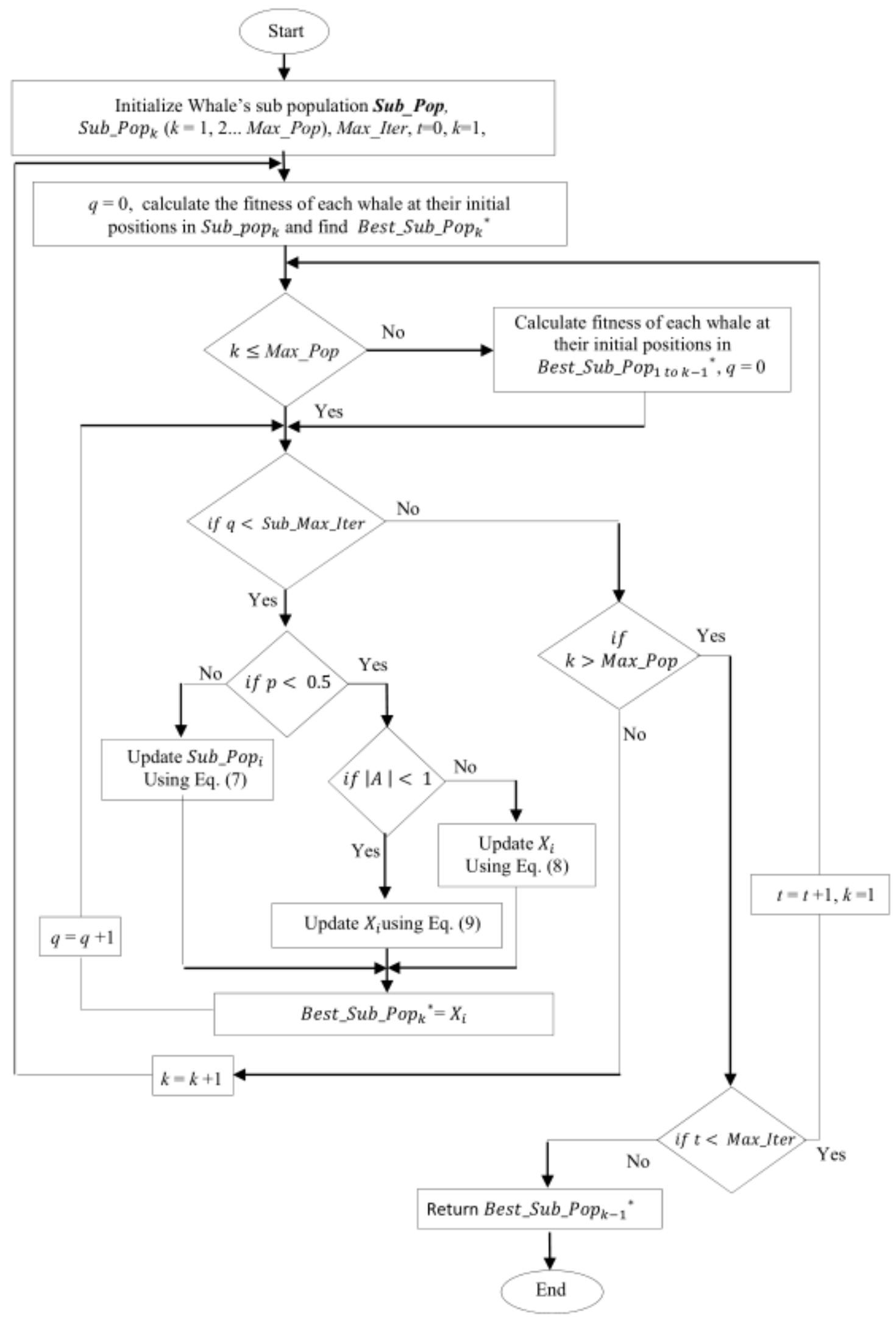

Fig.3. WOA invoking master-slave cooperative strategy 


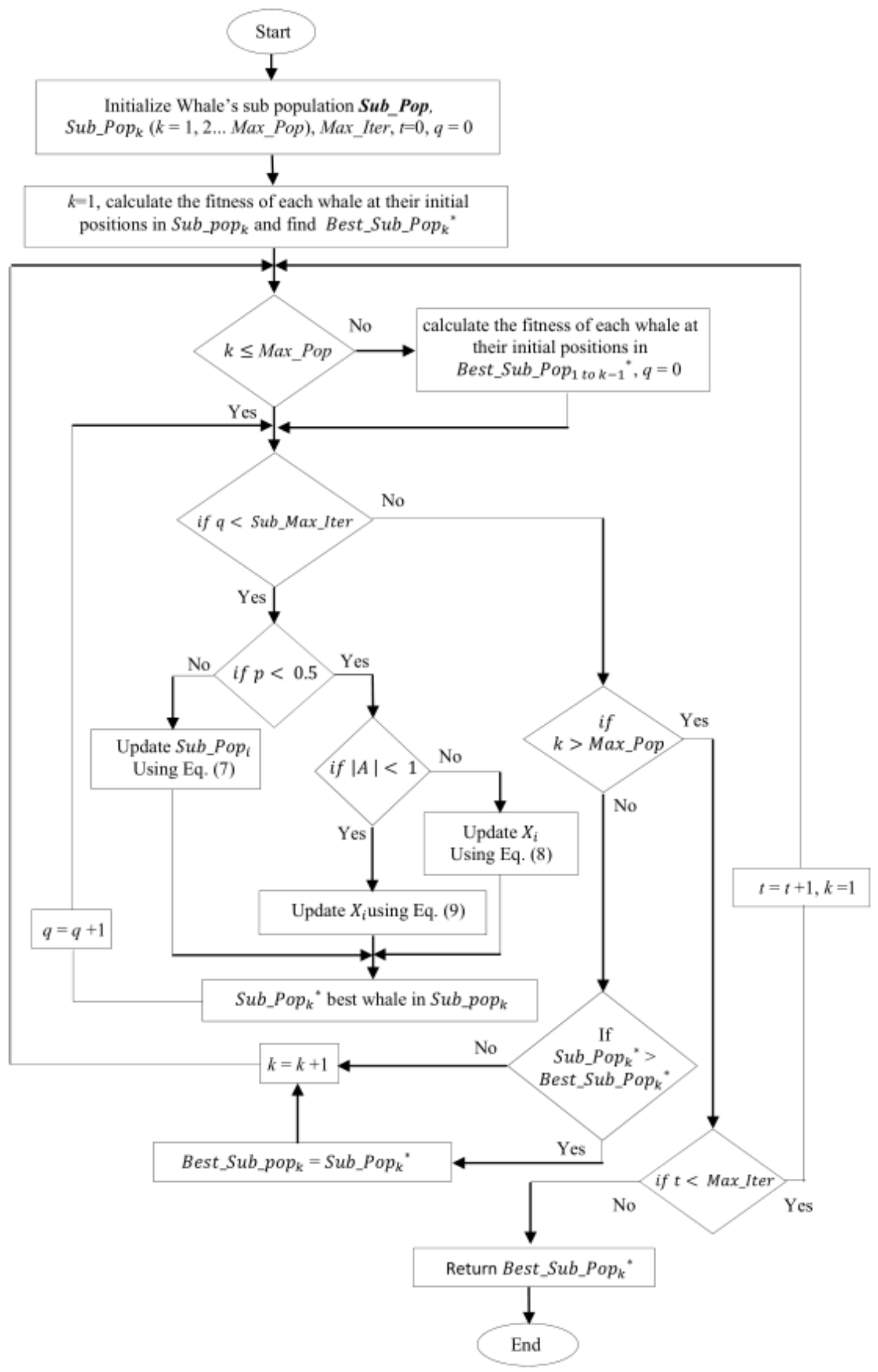

Fig.4. WOA invoking Hybrid cooperative strategy 
Table 4. Pseudo code for WOA invoking Master - Slave cooperative strategy

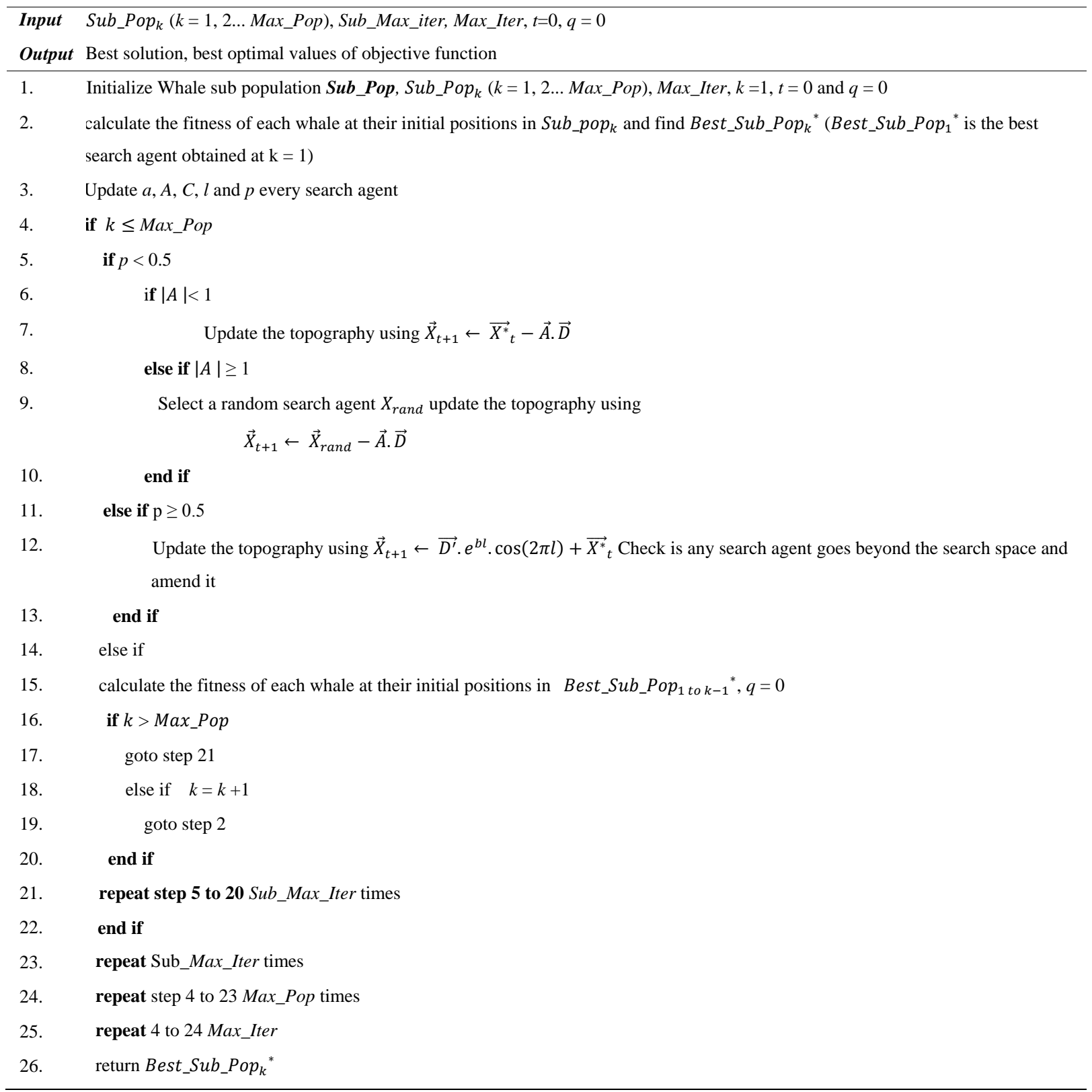

\section{EXPERIMENTAL RESULTS AND DISCUSSION}

In the current work, the performance of the proposed multi-swarm cooperative strategies (MsWOA) is compared with GSA, DE, PSO, WOA and variants of WOA in solving 23 standard mathematical benchmark functions and data clustering problems. We considered 10 real datasets for addressing the data clustering problems of which are taken from https://archive.ics.uci.edu. The detailed description of these datasets is listed in Table 6. In all the experiments, we have taken whales population of 30 and maximum of 500 iterations for both standard mathematical benchmark functions optimization problems and data clustering problems. All the conducted experiments and investigations have been made on an Intel(R) Core(TM)-i5-2400 CPU with a clock rate of 3.10 $\mathrm{GHz}, 8 \mathrm{~GB}$ RAM and proposed multi-swarm cooperative strategies for enhancing standard WOA is described earlier sections has been implemented using MATLABR2016a.

\section{A. Experiment 1: mathematical benchmark functions}

Like the other state-of-the-art and popular population based meta-heuristic optimization techniques such as GSA, DE [12], PSO [15], WOA [25] and advanced variants IWOA [26] and NPMOA [27] of WOA, makes use of a population to investigate the problem space. Population based mechanism bring into play the probability of obtaining optimal solution and abscond from local optima increases.

In this section, the proposed multi-swarm cooperative strategies for enhancing the optimization performance of standard WOA and compared the obtained results with GSA, DE, PSO, WOA, IWOA and NPMOA based on the 
optimal solutions obtained on 23 standard mathematical benchmark functions. We presented the comprehensive description of these benchmark functions in Table 1 a) and Table $1 \mathrm{~b}$ ). Note that the Table 1 a) indicates the comprehensive descriptions of 13 standard mathematical benchmark functions which include 7 Uni-modal and 6 Multimodal functions and Table 1 b) presents the description of 10 fixed dimension multimodal standard mathematical benchmark functions. The performance comparison have been made in terms of fitness convergence of GSA, DE, PSO, WOA and advanced variants IWOA and NPMOA of WOA and MsWOA for first four benchmark functions in graphical manner as given in Fig 5. The statistical analysis of obtained solution costs for MsWOA along with GSA, DE, PSO, WOA and variants of WOA is presented in a tabular form as given in Table 7. Hence, it is clear that the MsWOA is good at optimizes the most of the benchmark functions within a low cost solution than the other compared algorithms. Note that we used MsWOA for indicating all Ring (RWOA), Master-Slave (MSWOA) and Hybrid (HWOA) cooperative strategies in a single term.

Table 5. Pseudo code for WOA invoking Hybrid cooperative strategy

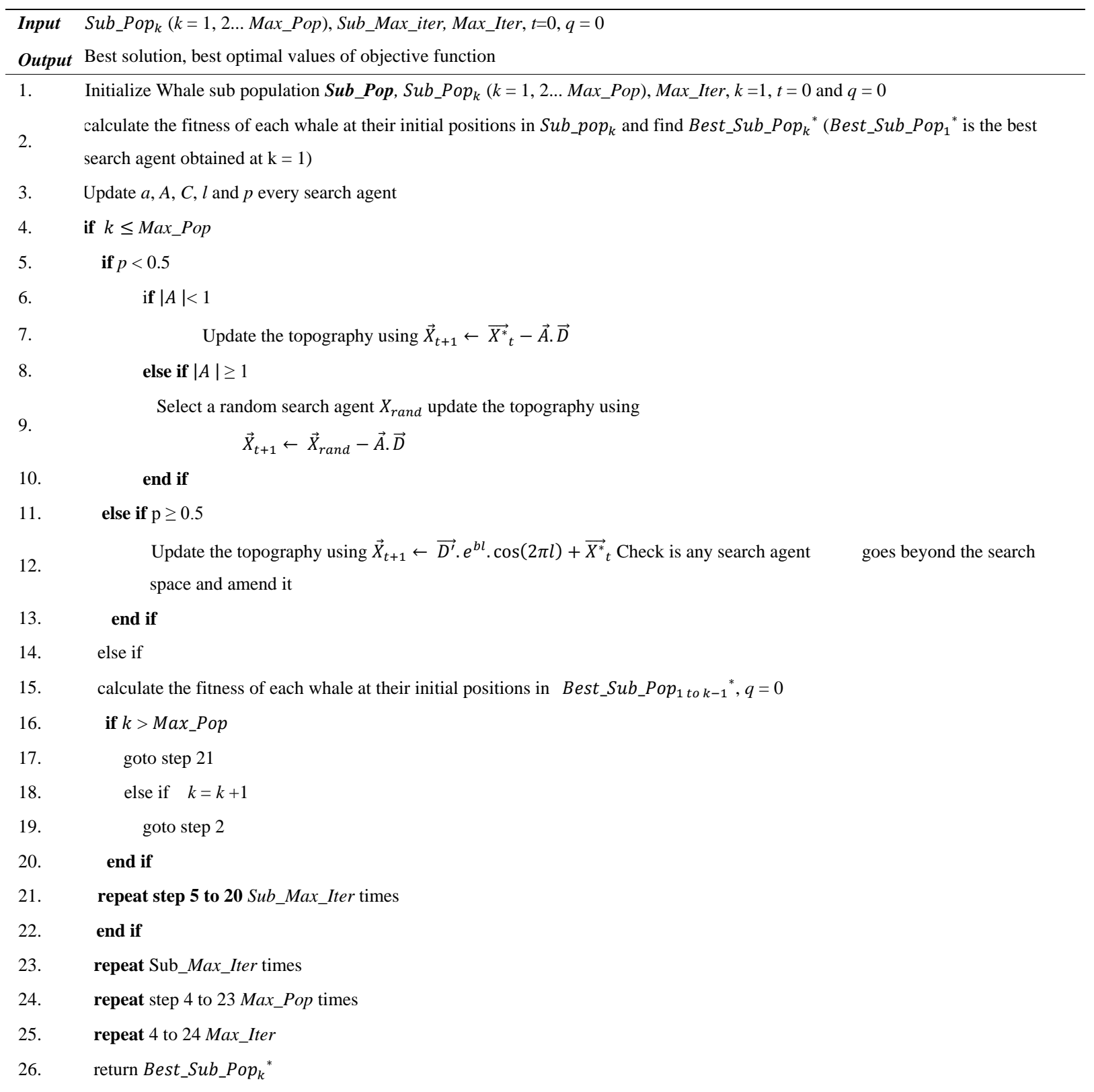

\section{B. Experiment 2: Data clustering problems}

To test the efficiency of MsWOA on data clustering problems, we examined 10 real datasets. The comprehensive descriptions of these datasets are displayed in Table 6. This section for the most part centered around data clustering using the proposed technique. At the point when a partitional clustering algorithm is said to be good, it should return optimal clusters in which the clustering strategy should indulge in minimization and maximization of intra cluster and inter cluster detachment respectively. The experimental and 
evidentical comparisons have been made with previously established algorithms. We used the fallowing three criteria to measure the clustering performance of each clustering technique. They are,

- $\quad$ Finding quantization error using Eq. (10)

- Intra-cluster minimization, i.e. where the key objective is to minimize the distance between data objects within a cluster.

- Inter-cluster maximization, i.e. where the key objective is to maximize the distance between the centroids of the clusters.

Quantization error $J_{e}=\frac{\sum_{j=1}^{N_{c}}\left[\sum_{\forall z_{p} \in C_{i, j}} d\left(z_{p}, m_{j}\right) /\left|c_{i, j}\right|\right]}{N_{c}}$
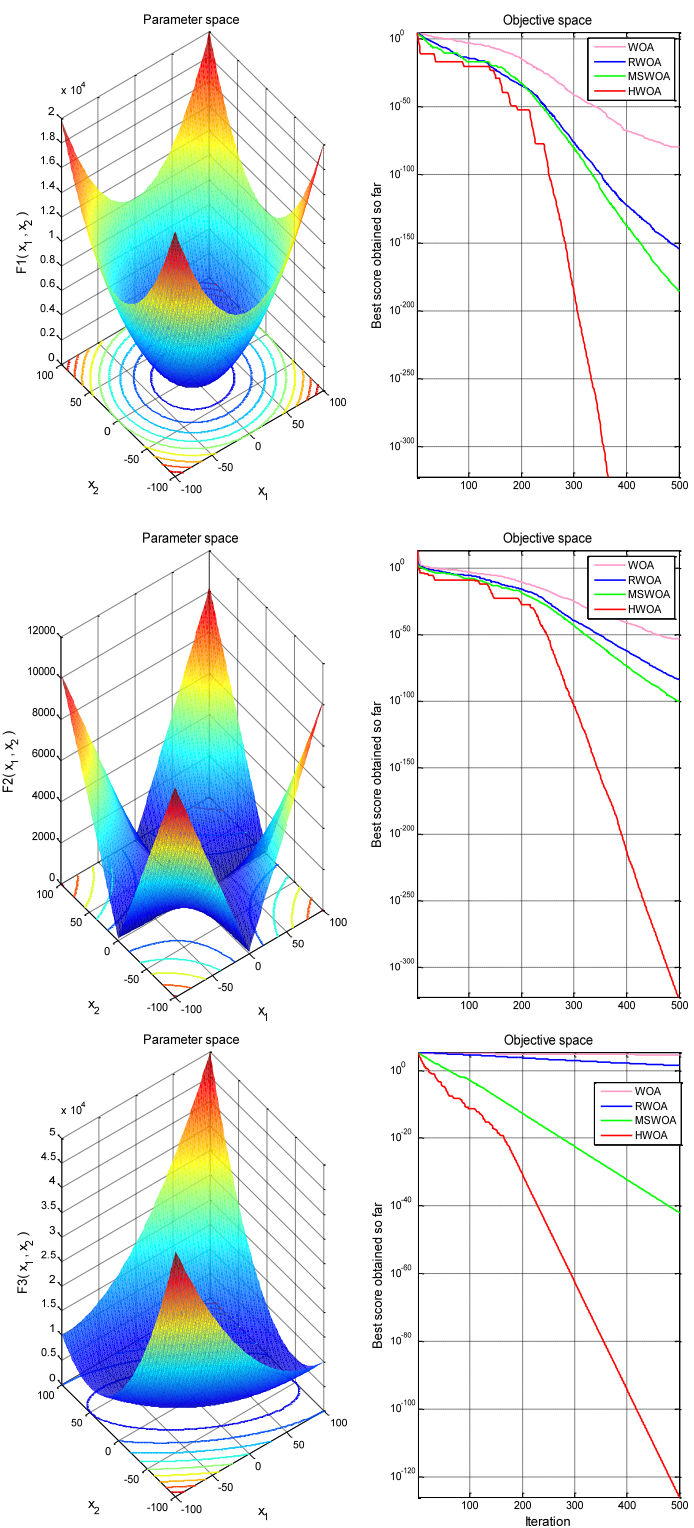
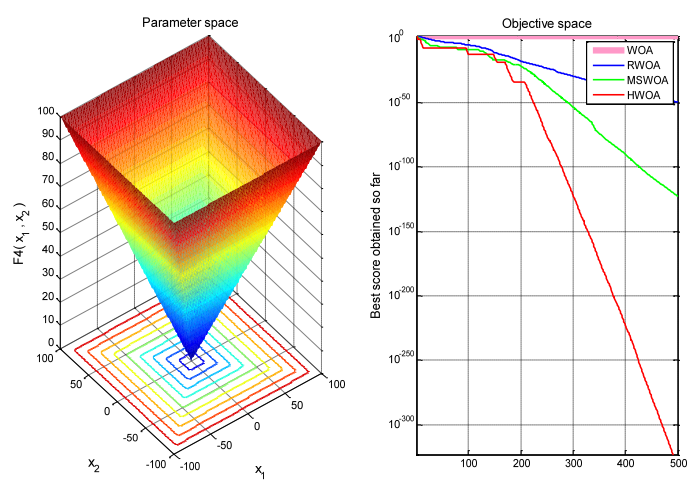

Fig.5. Fitness convergence of first four Uni-modal functions

Table 6. Description of datasets

\begin{tabular}{clccc}
\hline S. No & Dataset & size & dimension & $\boldsymbol{k}$ \\
\hline 1 & Iris & 150 & 4 & 3 \\
2 & Glass & 214 & 9 & 6 \\
3 & Wine & 178 & 13 & 3 \\
4 & Breast & 683 & 9 & 2 \\
5 & Pima Indian diabetes & 68 & 8 & 2 \\
6 & Haberman's survival & 306 & 3 & 2 \\
7 & Hayes-Roth & 160 & 4 & 3 \\
8 & E. Coli & 336 & 7 & 8 \\
9 & Zoo & 101 & 16 & 7 \\
10 & Vowel & 462 & 10 & 11 \\
\hline
\end{tabular}

In order to evaluate the MsWOA clustering performance, this algorithm is run on every benchmark dataset. From the obtained results the statistical analysis has been made. The convergence characteristics of data clustering problems are presented in a graphical manner in Fig.6. The mean and standard deviation are calculated for each test. The obtained solution cost, Intra-Cluster and Intra-Cluster distances for MsWOA are summarized in Table 8 along with previously established algorithms GSA, DE, PSO, WOA, IWOA, CSAWOA. In most of the cases the hybrid cooperative strategy improved the performance of WOA. From all the experiment results obtained for data clustering problems, we can testify that MsWOA works well in solving data clustering problems. Note that for all the data cluster problems, $k$ Means partitional clustering algorithm along with the standard Euclidean distance measuring technique is used. 
Table 7. Statistical analysis of obtained solution cost for $F_{1}$ to $F_{23}$

\begin{tabular}{|c|c|c|c|c|c|c|c|c|c|c|}
\hline \multirow{2}{*}{ Alg. } & \multicolumn{5}{|c|}{$F_{1}$} & \multicolumn{5}{|c|}{$\overline{F_{2}}$} \\
\hline & Average & Median & Std. Dev & Best & Worst & Average & Median & Std. Dev & Best & Worst \\
\hline GSA & $121.248 \mathrm{e}+01$ & $1.1549 \mathrm{e}-27$ & $8.1245 e+03$ & $1.1845 \mathrm{e}-51$ & $6.7843 e+04$ & $5.1532 \mathrm{e}+07$ & $2.1562 \mathrm{e}-09$ & $8.4238 \mathrm{e}+08$ & $3.4525 \mathrm{e}-47$ & $7.2637 \mathrm{e}+11$ \\
\hline DE & $132.328 \mathrm{e}+01$ & $1.4728 \mathrm{e}-27$ & $8.0715 e+03$ & $1.0548 \mathrm{e}-55$ & $6.9825 e+04$ & $5.4537 e+07$ & $2.5416 \mathrm{e}-09$ & $8.4751 \mathrm{e}+08$ & $3.9816 \mathrm{e}-47$ & $7.3519 \mathrm{e}+09$ \\
\hline PSO & $115.452 \mathrm{e}+01$ & $1.4528 \mathrm{e}-29$ & $8.0282 \mathrm{e}+03$ & $1.2457 \mathrm{e}-87$ & $6.8354 \mathrm{e}+04$ & $4.2193 e+07$ & $2.2023 \mathrm{e}-09$ & $8.7425 e+08$ & $3.5812 \mathrm{e}-47$ & $7.2581 \mathrm{e}+10$ \\
\hline WOA & 976.0925 & $1.4934 \mathrm{e}-29$ & $6.0966 \mathrm{e}+03$ & $1.0730 \mathrm{e}-81$ & $6.2422 \mathrm{e}+04$ & $2.9207 e+07$ & $1.7031 \mathrm{e}-18$ & $4.6134 \mathrm{e}+08$ & $3.4225 \mathrm{e}-52$ & $7.3017 \mathrm{e}+09$ \\
\hline IWOA & 141.0601 & $1.6987 \mathrm{e}-69$ & $2.8017 \mathrm{e}+03$ & $8.5331 \mathrm{e}-315$ & $6.2234 \mathrm{e}+04$ & $2.6816 \mathrm{e}+08$ & $1.1694 \mathrm{e}-32$ & $5.9961 \mathrm{e}+09$ & $2.1158 \mathrm{e}-159$ & $1.3408 \mathrm{e}+11$ \\
\hline CSAWOA & 143.4836 & $1.2152 \mathrm{e}-78$ & $3.2060 \mathrm{e}+03$ & 0 & $7.1689 \mathrm{e}+04$ & $1.0502 \mathrm{e}+06$ & $3.8957 \mathrm{e}-42$ & $2.3483 e+07$ & $1.1054 \mathrm{e}-202$ & $5.2510 \mathrm{e}+08$ \\
\hline RWOA & 278.4197 & $3.5175 e-53$ & $3.4636 \mathrm{e}+03$ & $3.0772 \mathrm{e}-155$ & $6.5930 \mathrm{e}+04$ & $1.1445 \mathrm{e}+09$ & $9.0683 \mathrm{e}-30$ & $2.5592 \mathrm{e}+10$ & $7.6042 \mathrm{e}-92$ & $5.7226 \mathrm{e}+11$ \\
\hline MSWOA & 148.2499 & $1.0370 \mathrm{e}-55$ & $2.1034 \mathrm{e}+03$ & $3.6666 \mathrm{e}-186$ & $4.2005 e+04$ & $6.2541 \mathrm{e}+08$ & $2.8133 \mathrm{e}-31$ & $1.3984 \mathrm{e}+10$ & $8.5322 \mathrm{e}-102$ & $3.1270 \mathrm{e}+11$ \\
\hline HWOA & 133.7060 & $3.1538 \mathrm{e}-94$ & $2.9709 \mathrm{e}+03$ & 0 & $6.6431 \mathrm{e}+04$ & $4.1330 \mathrm{e}+06$ & $1.0576 \mathrm{e}-49$ & $9.2418 \mathrm{e}+07$ & $5.5813 \mathrm{e}-318$ & $2.0665 e+09$ \\
\hline \multirow{2}{*}{ Alg. } & \multicolumn{5}{|c|}{$\overline{F_{3}}$} & \multicolumn{5}{|c|}{$F_{4}$} \\
\hline & Average & Median & Std. Dev & Best & Worst & Average & Median & Std. Dev & Best & Worst \\
\hline GSA & $5.2318 \mathrm{e}+05$ & $5.4532 \mathrm{e}+04$ & $3.8421 \mathrm{e}+05$ & $2.5136 \mathrm{e}+03$ & $1.3428 \mathrm{e}+05$ & 1.7672 & $2.4365 e-36$ & 5.6827 & $6.4162 \mathrm{e}-26$ & 112.3518 \\
\hline $\mathrm{DE}$ & $4.9845 e+05$ & $4.1429 \mathrm{e}+04$ & $3.5432 \mathrm{e}+05$ & $2.3684 \mathrm{e}+04$ & $1.3428 \mathrm{e}+05$ & 1.7516 & $2.6842 \mathrm{e}-36$ & 5.6845 & $6.6273 \mathrm{e}-26$ & 110.438 \\
\hline PSO & $4.7536 e+05$ & $4.2137 \mathrm{e}+04$ & $3.1452 \mathrm{e}+05$ & $2.2531 \mathrm{e}+04$ & $1.3549 \mathrm{e}+05$ & 1.2461 & $2.6821 \mathrm{e}-36$ & 5.3245 & $6.5736 \mathrm{e}-49$ & 100.2456 \\
\hline WOA & $5.7966 \mathrm{e}+04$ & $5.2965 \mathrm{e}+04$ & $3.4381 \mathrm{e}+04$ & $1.6992 \mathrm{e}+04$ & $1.2689 \mathrm{e}+05$ & 1.4157 & $2.2081 \mathrm{e}-35$ & 5.2690 & $6.3572 \mathrm{e}-38$ & 90.7160 \\
\hline IWOA & $1.2377 \mathrm{e}+03$ & $2.4741 \mathrm{e}-60$ & $1.6451 \mathrm{e}+04$ & $9.7553 e-270$ & $2.5731 \mathrm{e}+05$ & 0.2815 & $1.1628 \mathrm{e}-36$ & 4.2173 & $4.6036 \mathrm{e}-149$ & 87.9804 \\
\hline CSAWOA & 676.4248 & $7.3566 \mathrm{e}-74$ & $9.4417 e+03$ & $0.784 \mathrm{e}-270$ & $1.6777 \mathrm{e}+05$ & 0.2573 & $3.1044 \mathrm{e}-38$ & 4.2628 & $1.9567 \mathrm{e}-208$ & 86.0427 \\
\hline RWOA & $2.6761 \mathrm{e}+04$ & $2.0174 \mathrm{e}+03$ & $5.0740 \mathrm{e}+04$ & 30.4146 & $2.4953 e+05$ & 0.8732 & $6.0729 \mathrm{e}-24$ & 6.7354 & $2.3219 \mathrm{e}-50$ & 92.1388 \\
\hline MSWOA & $2.3047 \mathrm{e}+03$ & $2.8134 \mathrm{e}-18$ & $1.7782 \mathrm{e}+04$ & $1.1087 \mathrm{e}-42$ & $2.1653 e+05$ & 0.3378 & $1.8580 \mathrm{e}-37$ & 4.3973 & $1.5083 \mathrm{e}-123$ & 85.7551 \\
\hline HWOA & 635.1985 & $2.6796 \mathrm{e}-47$ & $8.1064 \mathrm{e}+03$ & $9.0514 \mathrm{e}-127$ & $1.5907 \mathrm{e}+05$ & 0.2516 & $1.4527 \mathrm{e}-76$ & 4.1991 & 0 & 87.8658 \\
\hline \multirow{2}{*}{ Alg. } & \multicolumn{5}{|c|}{$\mathbf{F}_{5}$} & \multicolumn{5}{|c|}{$F_{6}$} \\
\hline & Average & Median & Std. Dev & Best & Worst & Average & Median & Std. Dev & Best & Worst \\
\hline GSA & $2.9438 \mathrm{e}+06$ & 28.5925 & $1.7486 e+08$ & 29.8465 & $2.7165 e+08$ & 648.9124 & 0.4864 & $4.8462 \mathrm{e}+03$ & 0.4934 & $6.7168 \mathrm{e}+04$ \\
\hline $\mathrm{DE}$ & $2.7268 \mathrm{e}+06$ & 28.3516 & $1.7345 e+08$ & 29.5438 & $2.7465 e+08$ & 650.4671 & 0.45311 & $4.8513 e+03$ & 0.4271 & $6.4625 \mathrm{e}+04$ \\
\hline PSO & $2.8436 \mathrm{e}+06$ & 28.2816 & $1.7519 e+08$ & 29.1575 & $2.6427 e+08$ & 635.9215 & 0.3843 & $4.7364 \mathrm{e}+03$ & 0.4637 & $5.4928 \mathrm{e}+04$ \\
\hline WOA & $2.6080 \mathrm{e}+06$ & 27.9527 & $1.9373 e+07$ & 27.9516 & $2.5396 \mathrm{e}+08$ & 629.8011 & 0.3204 & $4.7976 \mathrm{e}+03$ & 0.3204 & $5.5280 \mathrm{e}+04$ \\
\hline IWOA & $4.0073 e+05$ & 27.8797 & $8.7116 \mathrm{e}+06$ & 27.8797 & $1.9476 \mathrm{e}+08$ & 168.6582 & 0.1528 & $3.2574 \mathrm{e}+03$ & 0.1528 & $7.2028 \mathrm{e}+04$ \\
\hline CSAWOA & $5.6167 \mathrm{e}+05$ & 28.6617 & $1.2501 \mathrm{e}+07$ & 28.6617 & $2.7952 \mathrm{e}+08$ & 135.4935 & 0.2798 & $2.8557 \mathrm{e}+03$ & 0.2798 & $6.3775 e+04$ \\
\hline RWOA & $6.4358 \mathrm{e}+05$ & 27.5877 & $1.0019 \mathrm{e}+07$ & 27.5877 & $2.1035 \mathrm{e}+08$ & 739.3369 & 0.3262 & $6.0110 \mathrm{e}+03$ & 0.3262 & $7.2705 \mathrm{e}+04$ \\
\hline MSWOA & $5.9859 \mathrm{e}+05$ & 27.9460 & $1.2597 \mathrm{e}+07$ & 27.9460 & $2.8119 \mathrm{e}+08$ & 607.4678 & 0.1720 & $4.6416 \mathrm{e}+03$ & 0.1720 & $6.1839 \mathrm{e}+04$ \\
\hline HWOA & $5.6736 \mathrm{e}+05$ & 28.4372 & $1.2686 \mathrm{e}+07$ & 28.4371 & $2.8366 \mathrm{e}+08$ & 158.9282 & 0.1634 & $2.7791 \mathrm{e}+03$ & 0.1633 & $6.0336 \mathrm{e}+04$ \\
\hline \multirow{2}{*}{ Alg. } & \multicolumn{5}{|c|}{$\mathbf{F}_{7}$} & & & $\mathbf{F}_{8}$ & & \\
\hline & Average & Median & Std. Dev & Best & Worst & Average & Median & Std. Dev & Best & Worst \\
\hline GSA & 2.6218 & 0.9261 & 12.9861 & 0.2897 & 150.6465 & $-1.2942 \mathrm{e}+04$ & $-1.5429 \mathrm{e}+04$ & $1.8435 \mathrm{e}+03$ & $-1.8415 e+04$ & $-3.4791 e+03$ \\
\hline DE & 2.6284 & 0.8649 & 12.8534 & 0.2785 & 150.6481 & $-1.2648 \mathrm{e}+04$ & $-1.6354 e+04$ & $1.7926 \mathrm{e}+03$ & $-1.7816 e+04$ & $-3.4627 e+03$ \\
\hline PSO & 1.6845 & 0.8634 & 12.6521 & 0.0654 & 148.5281 & $-1.2549 \mathrm{e}+04$ & $-1.4215 \mathrm{e}+04$ & $1.7924 \mathrm{e}+03$ & $-1.8195 e+04$ & $-3.4996 e+03$ \\
\hline WOA & 1.3233 & 0.0057 & 11.1443 & 0.0052 & 139.6725 & $-1.1491 e+04$ & $-1.2337 e+04$ & $1.7492 \mathrm{e}+03$ & $-1.2347 e+04$ & $-2.4467 e+03$ \\
\hline IWOA & 0.2960 & $2.3889 \mathrm{e}-04$ & 6.2970 & $4.0297 \mathrm{e}-05$ & 140.7692 & $-1.2393 e+04$ & $-1.2560 e+04$ & 897.0959 & $-1.2569 e+04$ & $-1.7542 e+03$ \\
\hline CSAWOA & 0.2358 & $8.3541 \mathrm{e}-04$ & 5.2301 & $9.0942 \mathrm{e}-05$ & 116.9492 & $-1.1683 e+04$ & $-1.2494 \mathrm{e}+04$ & $1.3604 \mathrm{e}+03$ & $-1.2569 \mathrm{e}+04$ & $-3.4988 \mathrm{e}+03$ \\
\hline RWOA & 0.2866 & $4.1339 \mathrm{e}-05$ & 6.3802 & $4.1339 \mathrm{e}-05$ & 142.6656 & $-1.1949 e+04$ & $-1.2569 e+04$ & $1.4897 \mathrm{e}+03$ & $-1.2569 e+04$ & $-2.2332 e+03$ \\
\hline MSWOA & 0.3021 & $4.5624 \mathrm{e}-04$ & 6.7172 & $7.0342 \mathrm{e}-05$ & 150.2019 & $-1.1731 \mathrm{e}+04$ & $-1.2430 \mathrm{e}+04$ & $1.5558 \mathrm{e}+03$ & $-1.2532 \mathrm{e}+04$ & $-2.2201 e+03$ \\
\hline HWOA & 0.1892 & $1.3918 \mathrm{e}-04$ & 4.2067 & $1.3918 \mathrm{e}-04$ & 94.0648 & $-1.1711 e+04$ & $-1.2201 \mathrm{e}+04$ & $1.3690 \mathrm{e}+03$ & $-1.2201 \mathrm{e}+04$ & $-1.5414 e+03$ \\
\hline A & & & $\overline{F_{9}}$ & & & & & $F_{10}$ & & \\
\hline Alg. & Average & Median & Std. Dev & Best & Worst & Average & Median & Std. Dev & Best & Worst \\
\hline GSA & 30.6527 & 0.2241 & 87.2954 & 0.0495 & 490.8134 & 1.2816 & $6.8435 \mathrm{e}-14$ & 2.9043 & $4.6205 \mathrm{e}-15$ & 20.6019 \\
\hline DE & 30.5468 & 0.2168 & 86.6218 & 0.0413 & 490.6218 & 1.1956 & $6.5269 \mathrm{e}-15$ & 3.0483 & $3.9352 \mathrm{e}-15$ & 20.7294 \\
\hline PSO & 30.2458 & 0.1762 & 86.1954 & 0.0361 & 485.1668 & 0.6583 & $6.6024 \mathrm{e}-15$ & 3.0201 & $4.6205 \mathrm{e}-15$ & 20.6903 \\
\hline WOA & 27.1213 & 0 & 76.7544 & 0 & 450.6246 & 0.4300 & $6.2172 \mathrm{e}-15$ & 2.5248 & $4.4409 \mathrm{e}-15$ & 20.4593 \\
\hline IWOA & 2.8204 & 0 & 28.9688 & 0 & 467.2299 & 0.1190 & $8.8818 \mathrm{e}-16$ & 1.2921 & $8.8818 \mathrm{e}-16$ & 20.8727 \\
\hline CSAWOA & 1.6330 & 0 & 24.0741 & 0 & 447.3239 & 0.1025 & $8.8818 \mathrm{e}-16$ & 1.2812 & $8.8818 \mathrm{e}-16$ & 20.6321 \\
\hline RWOA & 4.6452 & 0 & 35.8709 & 0 & 426.5025 & 0.2884 & $4.4409 \mathrm{e}-15$ & 1.9570 & $4.4409 \mathrm{e}-15$ & 20.7436 \\
\hline MSWOA & 0.9208 & 0 & 20.5897 & 0 & 460.3999 & 0.0420 & $8.8818 \mathrm{e}-16$ & 0.9380 & $8.8818 \mathrm{e}-16$ & 20.9750 \\
\hline HWOA & 1.4091 & 0 & 22.7304 & 0 & 425.1632 & 0.0448 & $8.8818 \mathrm{e}-16$ & 0.9233 & $8.8818 \mathrm{e}-16$ & 20.5905 \\
\hline A & & & $F_{11}$ & & & & & $F_{12}$ & & \\
\hline Alg. & Average & Median & Std. Dev & Best & Worst & Average & Median & Std. Dev & Best & Worst \\
\hline GSA & 7.1582 & 0 & 49.2604 & 0 & 615.1082 & $7.6207 e+06$ & 0.0486 & $5.7261 \mathrm{e}+07$ & 0.0486 & $7.4182 \mathrm{e}+08$ \\
\hline $\mathrm{DE}$ & 7.3518 & 0 & 49.1806 & 0 & 615.3409 & $7.0681 \mathrm{e}+06$ & 0.0482 & $5.8216 \mathrm{e}+07$ & 0.0482 & $7.9208 \mathrm{e}+08$ \\
\hline PSO & 6.9046 & 0 & 40.6608 & 0 & 614.7406 & $6.8106 e+06$ & 0.0465 & $5.6152 \mathrm{e}+07$ & 0.0465 & $7.8265 e+08$ \\
\hline WOA & 6.8496 & 0 & 51.1150 & 0 & 573.1051 & $6.5367 e+06$ & 0.0472 & $5.6013 e+07$ & 0.0472 & $7.4401 \mathrm{e}+08$ \\
\hline IWOA & 2.5364 & 0 & 37.8199 & 0 & 605.3266 & $1.7024 \mathrm{e}+06$ & 0.0101 & $3.1591 \mathrm{e}+07$ & 0.0101 & $6.8750 \mathrm{e}+08$ \\
\hline CSAWOA & 1.3268 & 0 & 29.4677 & 0 & 658.9239 & $1.4370 \mathrm{e}+06$ & 0.0073 & $3.2097 \mathrm{e}+07$ & 0.0073 & $7.1770 \mathrm{e}+08$ \\
\hline RWOA & 2.5074 & 0 & 31.0664 & 0 & 610.5245 & $9.9647 e+05$ & 0.0126 & $1.7356 \mathrm{e}+07$ & 0.0126 & $3.7500 \mathrm{e}+08$ \\
\hline MSWOA & 1.1732 & 0 & 25.8491 & 0 & 577.9697 & $1.3963 \mathrm{e}+06$ & 0.0149 & $3.1223 e+07$ & 0.0149 & $6.9817 \mathrm{e}+08$ \\
\hline HWOA & 1.1028 & 0 & 24.3672 & 0 & 501.3912 & $1.2914 \mathrm{e}+06$ & 0.0130 & $2.8878 \mathrm{e}+07$ & 0.0130 & $6.4572 \mathrm{e}+08$ \\
\hline
\end{tabular}


Table 7. Continued

\begin{tabular}{|c|c|c|c|c|c|c|c|c|c|c|}
\hline \multirow{2}{*}{ Alg. } & \multicolumn{5}{|c|}{$F_{13}$} & \multicolumn{5}{|c|}{$F_{14}$} \\
\hline & Average & Median & Std. Dev & Best & Worst & Average & Median & Std. Dev & Best & Worst \\
\hline GSA & $8.8435 \mathrm{e}+06$ & 0.4261 & $8.2696 e+07$ & 0.4261 & $3.4791 \mathrm{e}+08$ & 3.9406 & 2.9143 & 2.5492 & 2.9143 & 304.6271 \\
\hline DE & $9.4926 \mathrm{e}+06$ & 0.3158 & $8.8716 \mathrm{e}+07$ & 0.3158 & $3.4627 \mathrm{e}+08$ & 3.9603 & 2.9084 & 2.5834 & 2.9084 & 304.6419 \\
\hline PSO & $9.5109 e+06$ & 0.3151 & $8.3418 \mathrm{e}+07$ & 0.3151 & $3.4996 \mathrm{e}+08$ & 3.5038 & 2.9864 & 2.5681 & 2.9864 & 201.1934 \\
\hline WOA & $9.6103 e+06$ & 0.2720 & $8.2630 \mathrm{e}+07$ & 0.2720 & $9.5398 \mathrm{e}+08$ & 3.4340 & 2.9821 & 2.6263 & 2.9821 & 43.0259 \\
\hline IWOA & $2.5621 \mathrm{e}+06$ & 0.3602 & $5.6061 e+07$ & 0.3602 & $1.2533 \mathrm{e}+09$ & 1.8742 & 0.9980 & 18.6189 & 0.9980 & 416.9956 \\
\hline CSAWOA & $1.2597 \mathrm{e}+06$ & 0.1022 & $2.8129 \mathrm{e}+07$ & 0.1020 & $6.2899 \mathrm{e}+08$ & 1.3906 & 0.9980 & 5.3740 & 0.9980 & 115.8909 \\
\hline RWOA & $3.9986 \mathrm{e}+06$ & 0.2009 & $6.7180 e+07$ & 0.2008 & $1.4467 \mathrm{e}+09$ & 1.3106 & 0.9980 & 4.8911 & 0.9980 & 78.4131 \\
\hline MSWOA & $3.4805 \mathrm{e}+06$ & 0.2839 & $7.7827 \mathrm{e}+07$ & 0.2838 & $1.7403 e+09$ & 1.2186 & 0.9980 & 2.2341 & 0.9980 & 46.0861 \\
\hline HWOA & $2.1650 \mathrm{e}+06$ & 0.2324 & $4.8411 \mathrm{e}+07$ & 0.2323 & $1.0825 \mathrm{e}+09$ & 1.2154 & 0.9921 & 1.0419 & 0.9921 & 5.6662 \\
\hline \multirow{2}{*}{ Alg. } & \multicolumn{5}{|c|}{$F_{15}$} & \multicolumn{5}{|c|}{$\overline{F_{16}}$} \\
\hline & Average & Median & Std. Dev & Best & Worst & Average & Median & Std. Dev & Best & Worst \\
\hline GSA & 0.0072 & 0.0089 & 0.0348 & 0.0089 & 0.6428 & -1.0364 & -1.0316 & 0.2487 & -1.0316 & 0.1791 \\
\hline $\mathrm{DE}$ & 0.0086 & 0.0075 & 0.0365 & 0.0075 & 0.6612 & -1.0363 & -1.0316 & 0.2674 & -1.0316 & 0.1772 \\
\hline PSO & 0.0065 & 0.0081 & 0.0349 & 0.0081 & 0.5241 & -1.0316 & -1.0316 & 0.1642 & -1.0316 & 0.1541 \\
\hline WOA & 0.0015 & $3.2965 \mathrm{e}-04$ & 0.0205 & $3.2964 \mathrm{e}-04$ & 0.4502 & -1.0305 & -1.0316 & 0.0089 & -1.0316 & -0.8963 \\
\hline IWOA & $9.7449 \mathrm{e}-04$ & $4.6921 \mathrm{e}-04$ & 0.0038 & $4.6921 \mathrm{e}-04$ & 0.0746 & -1.0198 & -1.0316 & 0.1012 & -1.0316 & 0.1630 \\
\hline CSAWOA & $7.6984 \mathrm{e}-04$ & $3.1298 \mathrm{e}-04$ & 0.0056 & $3.1298 \mathrm{e}-04$ & 0.1238 & -1.0188 & -1.0316 & 0.1130 & -1.0316 & 1.0587 \\
\hline RWOA & 0.0016 & $4.9407 \mathrm{e}-04$ & 0.0210 & $4.9407 \mathrm{e}-04$ & 0.4707 & -0.9916 & -0.9999 & 0.1416 & -0.9999 & 2.1355 \\
\hline MSWOA & $6.6054 \mathrm{e}-04$ & $4.3257 \mathrm{e}-04$ & 0.0023 & $4.3257 \mathrm{e}-04$ & 0.0485 & -0.9878 & -0.9999 & 0.1989 & -0.9999 & 3.4022 \\
\hline HWOA & $6.0123 \mathrm{e}-04$ & $3.7381 \mathrm{e}-04$ & 0.0028 & $3.7258 \mathrm{e}-04$ & 0.0599 & -0.9931 & -1.0000 & 0.0350 & -1.0000 & -0.7368 \\
\hline \multirow{2}{*}{ Alg. } & \multicolumn{5}{|c|}{$F_{17}$} & \multicolumn{5}{|c|}{$F_{18}$} \\
\hline & Average & Median & Std. Dev & Best & Worst & Average & Median & Std. Dev & Best & \\
\hline GSA & 0.5247 & 0.3971 & 0.1736 & 0.3979 & 1.3601 & 3.4518 & 3.0000 & 3.0814 & 3.0000 & 34.4201 \\
\hline $\mathrm{DE}$ & 0.5716 & 0.3969 & 0.1705 & 0.3979 & 1.4608 & 3.5806 & 3.0000 & 3.0514 & 3.0000 & 34.1089 \\
\hline PSO & 0.4975 & 0.3951 & 0.1674 & 0.3979 & 2.3072 & 3.4841 & 3.0000 & 3.2153 & 3.0000 & 34.0045 \\
\hline WOA & 0.4256 & 0.3982 & 0.1260 & 0.3979 & 1.2673 & 3.5112 & 3.0000 & 3.5737 & 3.0000 & 34.9664 \\
\hline IWOA & 0.4115 & 0.3979 & 0.1003 & 0.3979 & 1.2713 & 3.3809 & 3.0000 & 3.0850 & 3.0000 & 33.3362 \\
\hline CSAWOA & 0.4000 & 0.3979 & 0.0155 & 0.3979 & 0.5795 & 3.0159 & 3.0000 & 0.1765 & 3.0000 & 5.7108 \\
\hline RWOA & 0.4324 & 0.3983 & 0.5675 & 0.3979 & 12.8981 & 3.1832 & 3.0184 & 1.3085 & 3.0183 & 22.4620 \\
\hline MSWOA & 0.4195 & 0.4024 & 0.0932 & 0.3985 & 1.0922 & 3.2130 & 3.0027 & 0.8532 & 3.0022 & 8.7739 \\
\hline HWOA & 0.4236 & 0.4001 & 0.1144 & 0.3999 & 1.6499 & 3.7103 & 3.0000 & 5.2395 & 3.0000 & 79.2618 \\
\hline \multirow{2}{*}{ Alg. } & \multicolumn{5}{|c|}{$F_{19}$} & & & $F_{20}$ & & \\
\hline & Average & Median & Std. Dev & Best & Worst & Average & Median & Std. Dev & Best & Worst \\
\hline GSA & -3.8514 & -3.8567 & 0.0721 & -3.8587 & -3.4802 & -3.2142 & -2.0701 & 0.0618 & -3.1014 & -1.7692 \\
\hline DE & -3.8587 & -3.8712 & 0.0506 & -3.8654 & -2.9634 & -2.8401 & -2.4602 & 0.0681 & -3.0285 & -1.5593 \\
\hline PSO & -3.8297 & -3.8409 & 0.0501 & -3.8542 & -3.2409 & -3.2406 & -3.0019 & 0.0605 & -1.4018 & -1.4608 \\
\hline WOA & -3.8218 & -3.8560 & 0.0616 & -3.8565 & -3.4609 & -2.9480 & -2.9899 & 0.0695 & -2.9900 & -2.3866 \\
\hline IWOA & -3.8585 & -3.8613 & 0.0411 & -3.8614 & -2.9634 & -3.2169 & -3.3014 & 0.1696 & -3.3015 & -1.7692 \\
\hline CSAWOA & -3.8290 & -3.8549 & 0.0429 & -3.8549 & -3.2802 & -2.9522 & -3.0017 & 0.1452 & -3.0235 & -1.5593 \\
\hline RWOA & -3.7960 & -3.8249 & 0.0552 & -3.8260 & -3.3592 & -3.2815 & -3.3143 & 0.1464 & -3.3143 & -1.5171 \\
\hline MSWOA & -3.8128 & -3.8612 & 0.0725 & -3.8612 & -3.5227 & -2.6257 & -2.6756 & 0.2733 & -2.6756 & -0.8829 \\
\hline HWOA & -3.8057 & -3.8553 & 0.1308 & -3.8553 & -3.0619 & -3.0471 & -3.1437 & 0.2353 & -3.1437 & -1.4009 \\
\hline $\mathbf{A l g}$ & & & $F_{21}$ & & & & & $F_{22}$ & & \\
\hline Alg. & Average & Median & Std. Dev & Best & Worst & Average & Median & Std. Dev & Best & Worst \\
\hline GSA & -3.9021 & -4.5407 & 2.0154 & -3.1408 & -0.9154 & -2.1008 & -3.8560 & 0.3144 & -3.0019 & -0.4102 \\
\hline DE & -3.7463 & -4.3518 & 2.4106 & -3.9215 & -0.9425 & -3.1473 & -3.0417 & 0.4935 & -2.9480 & -0.5657 \\
\hline PSO & -4.4026 & -4.0407 & 0.3109 & -4.2831 & -9.7106 & -3.4083 & -3.8409 & 0.2954 & -2.9900 & -0.9142 \\
\hline WOA & -4.9423 & -5.0547 & 0.4996 & -5.0547 & -0.5249 & -3.6383 & -3.7213 & 0.3054 & -3.7213 & -0.5457 \\
\hline IWOA & -9.9569 & -10.0979 & 0.8079 & -10.1128 & -2.0944 & -6.3471 & -6.8573 & 0.8925 & -6.8695 & -1.0269 \\
\hline CSAWOA & -6.9943 & -5.0521 & 2.5053 & -9.9571 & -0.4070 & -8.1818 & -9.4109 & 1.9301 & -9.4130 & -0.5524 \\
\hline RWOA & -5.0086 & -5.0528 & 0.3067 & -5.0529 & -0.4786 & -9.3501 & -10.3475 & 1.9994 & -10.3630 & -0.7678 \\
\hline MSWOA & -4.5664 & -4.6989 & 0.2979 & -4.6989 & -0.4279 & -3.3935 & -3.8828 & 0.8519 & -3.8828 & -0.4594 \\
\hline HWOA & -4.4230 & -4.5876 & 0.4779 & -4.5876 & -0.3629 & -4.9339 & -4.9794 & 0.3872 & -4.9794 & -0.3981 \\
\hline $\mathrm{Alo}$ & & & $F_{23}$ & & & & & & & \\
\hline Alg. & Average & Median & Std. Dev & Best & Worst & & & & & \\
\hline GSA & -1.9215 & -4.0019 & 0.4512 & -0.8963 & -0.9142 & & & & & \\
\hline $\mathrm{DE}$ & -1.1408 & -3.4602 & 0.4654 & -0.1541 & -1.5893 & & & & & \\
\hline PSO & -1.2831 & -4.5593 & 0.4171 & -0.1630 & -1.5593 & & & & & \\
\hline WOA & -5.0517 & -5.1273 & 0.3852 & -5.1273 & -1.3220 & & & & & \\
\hline IWOA & -2.3074 & -2.4192 & 0.3146 & -2.4192 & -0.6555 & & & & & \\
\hline CSAWOA & -5.5784 & -5.1016 & 1.5542 & -9.0782 & -0.8072 & & & & & \\
\hline RWOA & -2.3955 & -2.4244 & 0.1182 & -2.4251 & -0.7247 & & & & & \\
\hline MSWOA & -3.8022 & -4.0244 & 0.5487 & -4.0244 & -0.6114 & & & & & \\
\hline HWOA & -4.7822 & -5.0209 & 0.6715 & -5.0209 & -1.1131 & & & & & \\
\hline
\end{tabular}


Table 8. Mean and std. dev. over 30 independent runs for 500 iterations for 10 real datasets

\begin{tabular}{|c|c|c|c|c|c|c|}
\hline \multirow{2}{*}{ Alg. } & \multicolumn{3}{|c|}{ Iris } & \multicolumn{3}{|c|}{ Glass } \\
\hline & Solution cost & Intra-cluster & Inter-cluster & Solution cost & Intra-cluster & Inter-cluster \\
\hline$\overline{\text { GSA }}$ & $0.2791 \pm 0.0157$ & $2.4925 \pm 0.3709$ & $1.9247 \pm 0.2746$ & $0.0565 \pm 0.0049$ & $5.2019 \pm 0.8524$ & $5.2634 \pm 6.4387$ \\
\hline $\mathrm{DE}$ & $0.2787 \pm 0.0151$ & $2.2405 \pm 0.3918$ & $1.9219 \pm 0.2778$ & $0.0584 \pm 0.0027$ & $5.2113 \pm 0.8509$ & $4.7738 \pm 9.2009$ \\
\hline PSO & $0.2787 \pm 0.0152$ & $2.4703 \pm 0.3725$ & $1.9701 \pm 0.2784$ & $0.0428 \pm 0.0047$ & $5.0216 \pm 0.7034$ & $6.0021 \pm 7.3475$ \\
\hline WOA & $0.2672 \pm 0.0141$ & $2.3902 \pm 0.3562$ & $1.9291 \pm 0.2789$ & $0.0443 \pm 0.0042$ & $5.0199 \pm 0.7014$ & $4.7812 \pm 9.2109$ \\
\hline IWOA & $0.2765 \pm 0.0042$ & $2.2258 \pm 0.3601$ & $1.9121 \pm 0.2821$ & $0.0436 \pm 0.0027$ & $4.9542 \pm 0.5987$ & $5.2514 \pm 6.4521$ \\
\hline CSAWOA & $0.2681 \pm 0.0071$ & $2.2258 \pm 0.3429$ & $1.9623 \pm 0.2731$ & $0.0435 \pm 0.0029$ & $4.8781 \pm 0.8924$ & $6.0081 \pm 7.3125$ \\
\hline RWOA & $0.2689 \pm 9.5741 \mathrm{e}-004$ & $2.1222 \pm 0.3522$ & $1.9802 \pm 0.2189$ & $0.0433 \pm 0.0017$ & $4.8745 \pm 0.8957$ & $6.4189 \pm 6.9702$ \\
\hline MSWOA & $0.2655 \pm 0.0067$ & $2.0641 \pm 0.3434$ & $1.9291 \pm 0.2789$ & $0.0429 \pm 0.0031$ & $4.0934 \pm 0.7134$ & $6.7204 \pm 9.0704$ \\
\hline HWOA & $0.2645 \pm 0.0064$ & $2.0176 \pm 0.3427$ & $1.9802 \pm 0.2189$ & $0.0414 \pm 0.0014$ & $4.3705 \pm 0.7447$ & $6.7612 \pm 6.4308$ \\
\hline \multirow{2}{*}{ Alg. } & \multicolumn{3}{|c|}{ Wine } & \multicolumn{3}{|c|}{ Breast } \\
\hline & Solution cost & Intra-cluster & Inter-cluster & Solution cost & Intra-cluster & Inter-cluster \\
\hline$\overline{\text { GSA }}$ & $360.8613 \pm 1.8143$ & $364.6584 \pm 8.6347$ & $328.5411 \pm 3.4305$ & $0.0672 \pm 0.0214$ & $17.1289 \pm 3.7189 \mathrm{e}-015$ & $13.4628 \pm 0$ \\
\hline $\mathrm{DE}$ & $359.4155 \pm 11.1054$ & $362.0013 \pm 9.8672$ & $359.8273 \pm 54.4602$ & $0.0621 \pm 0.0275$ & $16.9721 \pm 7.46 \mathrm{e}-15$ & $13.4654 \pm 0$ \\
\hline PSO & $343.8378 \pm 3.5681$ & $350.1967 \pm 7.5448$ & $349.7465 \pm 46.7305$ & $0.0563 \pm 0.0361$ & $17.0346 \pm 8.01 \mathrm{e}-15$ & $13.5438 \pm 0$ \\
\hline WOA & $340.1125 \pm 10.9298$ & $350.1951 \pm 7.5439$ & $329.2461 \pm 71.9199$ & $0.0561 \pm 0.0285$ & $17.0246 \pm 7.95 \mathrm{e}-15$ & $13.4529 \pm 0$ \\
\hline IWOA & $340.8713 \pm 1.7802$ & $341.7484 \pm 8.6213$ & $329.2701 \pm 72.4532$ & $0.0552 \pm 0.0128$ & $17.0238 \pm 8.01 \mathrm{e}-15$ & $13.4536 \pm 0$ \\
\hline CSAWOA & $342.8378 \pm 3.6219$ & $341.0013 \pm 9.8928$ & $363.7845 \pm 46.7197$ & $0.0546 \pm 0.0341$ & $17.0238 \pm 7.89 \mathrm{e}-15$ & $13.4523 \pm 0$ \\
\hline RWOA & $341.7219 \pm 6.8014 \mathrm{e}-014$ & $332.7921 \pm 2.2318$ & $359.8712 \pm 52.3245$ & $0.0551 \pm 6.7872 \mathrm{e}-004$ & $16.9804 \pm 7.45 \mathrm{e}-15$ & $13.5521 \pm 0$ \\
\hline MSWOA & $341.6293 \pm 1.6702$ & $330.1041 \pm 7.4414$ & $345.2121 \pm 47.3042$ & $0.0547 \pm 0.1385$ & $16.0426 \pm 7.75 \mathrm{e}-15$ & $13.4521 \pm 0$ \\
\hline HWOA & $339.6378 \pm 1.6716$ & $330.1181 \pm 7.4034$ & $329.2711 \pm 47.9159$ & $0.0534 \pm 0.0234$ & $16.4036 \pm 7.71 \mathrm{e}-15$ & $13.4520 \pm 0$ \\
\hline \multirow{2}{*}{ Alg. } & \multicolumn{3}{|c|}{ Diabetes } & \multicolumn{3}{|c|}{ Haberman } \\
\hline & Solution cost & Intra-cluster & Inter-cluster & Solution cost & Intra-cluster & Inter-cluster \\
\hline$\overline{\text { GSA }}$ & $14.5400 \pm 0.1048$ & $730.0298 \pm 0.1502$ & $30.4512 \pm 0.4152$ & $5.4800 \pm 0.3273$ & $40.3214 \pm 2.1089 \mathrm{e}-014$ & $5.2185 \pm 8.8425 \mathrm{e}-016$ \\
\hline $\mathrm{DE}$ & $14.5701 \pm 0.1814$ & $713.6642 \pm 74.3624$ & $36.2192 \pm 12.2457$ & $5.5629 \pm 0.2713$ & $35.1085 \pm 3.5591$ & $8.7361 \pm 10.8134$ \\
\hline PSO & $14.4416 \pm 0.0548$ & $713.6502 \pm 74.3512$ & $36.2192 \pm 12.2401$ & $5.7000 \pm 0.3045$ & $35.3451 \pm 3.6982$ & $7.3547 \pm 9.8732$ \\
\hline WOA & $14.2800 \pm 0.1654$ & $708.0127 \pm 74.4521$ & $36.2301 \pm 14.7728$ & $5.7700 \pm 0.2845$ & $35.3026 \pm 3.6534$ & $7.6424 \pm 9.8043$ \\
\hline IWOA & $13.8000 \pm 0.0954$ & $707.0087 \pm 74.6214$ & $36.2354 \pm 14.7602$ & $5.7800 \pm 0.3215$ & $35.2445 \pm 3.784$ & $7.9401 \pm 9.9731$ \\
\hline CSAWOA & $13.8524 \pm 0.0459$ & $705.9815 \pm 74.3714$ & $36.8004 \pm 14.4543$ & $5.3300 \pm 0.3545$ & $35.2064 \pm 3.0475$ & $7.9821 \pm 9.9801$ \\
\hline RWOA & $13.7689 \pm 0.0025$ & $705.9802 \pm 74.3512$ & $36.8425 \pm 14.4512$ & $5.5689 \pm 3.6142 \mathrm{e}-006$ & $34.9885 \pm 3.6875$ & $8.1285 \pm 10.4219$ \\
\hline MSWOA & $13.7741 \pm 0.0454$ & $705.0164 \pm 74.6151$ & $36.3051 \pm 14.3508$ & $5.3655 \pm 0.3065$ & $34.3721 \pm 3.5504$ & $7.3047 \pm 9.8042$ \\
\hline HWOA & $13.7453 \pm 0.0656$ & $705.0045 \pm 74.3402$ & $36.3801 \pm 14.3461$ & $5.2814 \pm 0.3061$ & $34.3125 \pm 3.5530$ & $7.3607 \pm 9.8342$ \\
\hline \multirow{2}{*}{ Alg. } & \multicolumn{3}{|c|}{ Hayes-Roth } & \multicolumn{3}{|c|}{ E. Coli } \\
\hline & Solution cost & Intra-cluster & Inter-cluster & Solution cost & Intra-cluster & Inter-cluster \\
\hline$\overline{\text { GSA }}$ & $0.3279 \pm 0.0324$ & $3.5643 \pm 0.4428$ & $1.3915 \pm 0.3541$ & $0.0672 \pm 0.0373$ & $0.6648 \pm 0.0834$ & $0.2445 \pm 0.0598$ \\
\hline $\mathrm{DE}$ & $0.3928 \pm 0.0346$ & $3.518 \pm 0.2735$ & $1.8519 \pm 0.7531$ & $0.0663 \pm 0.0032$ & $0.6643 \pm 0.0735$ & $0.3637 \pm 0.1341$ \\
\hline PSO & $0.3507 \pm 0.0254$ & $3.5081 \pm 0.4318$ & $1.4189 \pm 0.6078$ & $0.0671 \pm 0.0030$ & $0.6312 \pm 0.0726$ & $0.3145 \pm 0.1028$ \\
\hline WOA & $0.3672 \pm 0.0284$ & $3.5492 \pm 0.4865$ & $1.4256 \pm 0.6845$ & $0.0641 \pm 0.0028$ & $0.6745 \pm 0.0825$ & $0.3176 \pm 0.1517$ \\
\hline IWOA & $0.3765 \pm 0.0251$ & $3.5489 \pm 0.4812$ & $1.4364 \pm 0.6704$ & $0.0672 \pm 0.0028$ & $0.6545 \pm 0.0754$ & $0.3522 \pm 0.1102$ \\
\hline CSAWOA & $0.2681 \pm 0.0248$ & $3.5413 \pm 0.4716$ & $1.7012 \pm 0.6146$ & $0.0666 \pm 0.0030$ & $0.6245 \pm 0.0689$ & $0.4540 \pm 0.1056$ \\
\hline RWOA & $0.2689 \pm 0.0129$ & $3.2508 \pm 0.3512$ & $1.8251 \pm 0.6702$ & $0.0641 \pm 0.0012$ & $0.6012 \pm 0.0542$ & $0.4643 \pm 0.1728$ \\
\hline MSWOA & $0.2655 \pm 0.0105$ & $3.3415 \pm 0.4181$ & $1.4046 \pm 0.6451$ & $0.0637 \pm 0.00124$ & $0.6015 \pm 0.0621$ & $0.3206 \pm 0.1410$ \\
\hline HWOA & $0.2645 \pm 0.0108$ & $3.5472 \pm 0.4160$ & $1.4053 \pm 0.6415$ & $0.0624 \pm 0.00113$ & $0.6018 \pm 0.0721$ & $0.3603 \pm 0.1421$ \\
\hline \multirow{2}{*}{ Alg. } & \multicolumn{3}{|c|}{ Zoo } & \multicolumn{3}{|c|}{ Vowel } \\
\hline & Solution cost & Intra-cluster & Inter-cluster & Solution cost & Intra-cluster & Inter-cluster \\
\hline$\overline{\text { GSA }}$ & $0.0202 \pm 0.0026$ & $1.6378 \pm 1.1528 \mathrm{e}-15$ & $2.2315 \pm 5.0214 \mathrm{e}-016$ & $41.9500 \pm 1.3465$ & $901.3251 \pm 108.8745$ & $348.3268 \pm 230.3519$ \\
\hline $\mathrm{DE}$ & $0.0220 \pm 0.0024$ & $1.6512 \pm 1.1324 \mathrm{e}-15$ & $2.6502 \pm 2.278 \mathrm{e}-15$ & $39.2800 \pm 2.2481$ & $898.617 \pm 102.9459$ & $353.3712 \pm 218.2482$ \\
\hline PSO & $0.0130 \pm 0.0018$ & $1.6396 \pm 1.1244 \mathrm{e}-15$ & $2.6421 \pm 2.256 \mathrm{e}-15$ & $38.4700 \pm 2.3618$ & $899.254 \pm 101.8954$ & $350.3465 \pm 215.7135$ \\
\hline WOA & $0.0120 \pm 0.0022$ & $1.6401 \pm 1.1312 \mathrm{e}-15$ & $2.6471 \pm 2.274 \mathrm{e}-15$ & $38.4012 \pm 2.2314$ & $899.246 \pm 101.8705$ & $350.3548 \pm 215.6548$ \\
\hline IWOA & $0.0110 \pm 0.0021$ & $1.6391 \pm 1.1268 \mathrm{e}-15$ & $2.6484 \pm 2.25 \mathrm{e}-15$ & $37.6824 \pm 1.3545$ & $899.238 \pm 102.3254$ & $350.7091 \pm 215.9805$ \\
\hline CSAWOA & $0.0090 \pm 0.0019$ & $1.6381 \pm 1.1254 \mathrm{e}-15$ & $2.6487 \pm 2.258 \mathrm{e}-15$ & $36.8140 \pm 2.3456$ & $899.228 \pm 102.3815$ & $350.8086 \pm 215.9501$ \\
\hline RWOA & $0.0094 \pm 8.3665-04$ & $1.5254 \pm 4.5498 \mathrm{e}-016$ & $2.6502 \pm 2.256 \mathrm{e}-15$ & $35.8941 \pm 2.2145$ & $898.145 \pm 102.9821$ & $353.3282 \pm 218.2545$ \\
\hline MSWOA & $0.0100 \pm 0.0012$ & $1.5332 \pm 1.1268 \mathrm{e}-14$ & $2.6451 \pm 2.264 \mathrm{e}-15$ & $34.1553 \pm 2.2645$ & $898.224 \pm 102.3142$ & $350.7301 \pm 215.9611$ \\
\hline HWOA & $0.00534 \pm 0.0024$ & $1.4301 \pm 1.1341 \mathrm{e}-14$ & $2.6432 \pm 2.261 \mathrm{e}-15$ & $32.4501 \pm 2.2416$ & $898.138 \pm 102.3107$ & $350.6126 \pm 215.9411$ \\
\hline
\end{tabular}



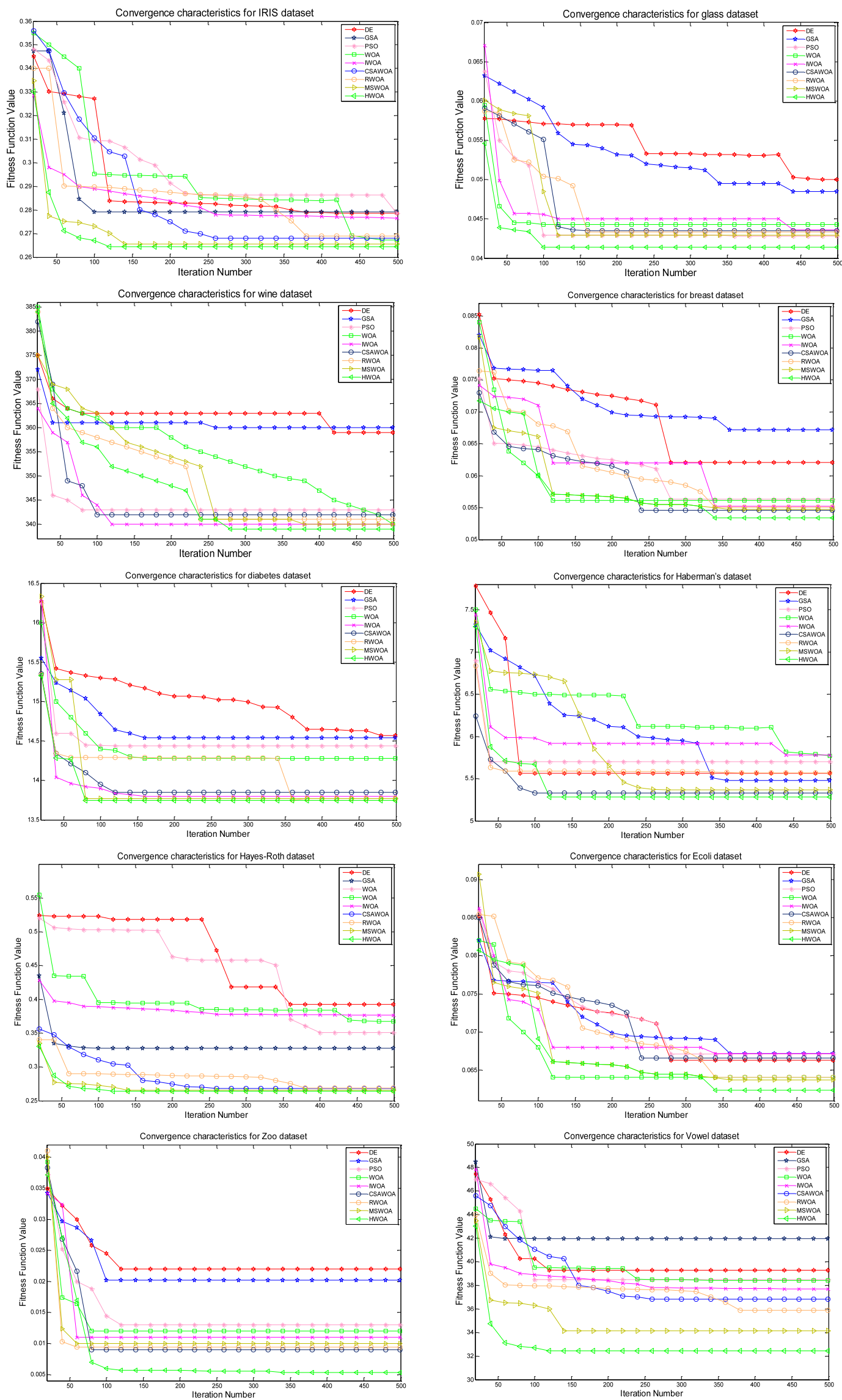

Fig.6. Convergence of data clustering problems 


\section{CONCLUSIONS AND FUTURE WORK}

In this work we presented multi-swarm cooperative strategies for addressing two problems. They are solving standard mathematical benchmark functions problems and solving the data clustering problems. In this process we have used the standard whale optimization algorithm. We utilized three cooperative strategies namely the Ring, Master-Slave and Hybrid cooperative strategies to enhance the performance of standard WOA. Several experiments have been conducted and the performance of each cooperative strategy is compared with previously established techniques GSA, DE, PSO, WOA and also variants of WOA. The results established the multi-swarm cooperative strategies outperform in solving benchmark functions as well as the data clustering problems compared GSA, DE, PSO, WOA and variants of WOA for 10 different real databases. All the obtained graphical and statistical results are reported respective figures and tables. The reported values were averaged over 40 simulations to specify the algorithms convergence range. In most cases, the proposed multi-swarm cooperative strategies realized lower quantization errors. Generally, the proposed multi-swarm cooperative strategies establish their efficiency in finding optimizing benchmark functions as well as the data clustering problems.

This explore run may be extended for further research in the shadowing dimensions: In this research work, the show on multiple swarm based cooperative strategies proposed for enhancing the numerical optimization performance of WOA and these are compared with other-state-of-the-art algorithms and variants of WOA for its action judgement on 23 standard benchmark functions. As a future line, added new versions of WOA could also be tried to key the unexceeded type in achieving test suite improvement. This research work concentrate on standard WOA optimization. In the future we go through the new population based meta-heuristic optimization algorithms which are also inspired by the nature.

\section{REFERENCES}

[1] Kacprzyk, Janusz, and Witold Pedrycz, eds. Springer handbook of computational intelligence. Springer, 2015.

[2] Amiri, Babak. "Application of teaching-learning-based optimization algorithm on cluster analysis." Journal of Basic and Applied Scientific Research 2.11 (2012): 1179511802.

[3] Hoang, Duc Chinh, et al. "Real-time implementation of a harmony search algorithm-based clustering protocol for energy-efficient wireless sensor networks." IEEE Transactions on Industrial Informatics 10.1 (2014): $774-$ 783.

[4] Kuo, R. J., et al. "Integration of particle swarm optimization and genetic algorithm for dynamic clustering." Information Sciences 195 (2012): 124-140.

[5] Niknam, Taher, et al. "An efficient hybrid evolutionary optimization algorithm based on PSO and SA for clustering." Journal of Zhejiang University-SCIENCE A 10.4 (2009): 512-519.
[6] Nanda, Satyasai Jagannath, and Ganapati Panda. "A survey on nature inspired metaheuristic algorithms for partitional clustering." Swarm and Evolutionary computation 16 (2014): 1-18.

[7] Holland JH . Genetic algorithms. Sci Am 1992;267:66-72.

[8] Rahman, Md Anisur, and Md Zahidul Islam. "A hybrid clustering technique combining a novel genetic algorithm with K-Means." Knowledge-Based Systems 71 (2014): 345-365.

[9] Dorigo M, Gambardella LM (1997) Ant colony system: a cooperative learning approach to the traveling salesman problem. IEEE Trans Evol Comput 12:53-66.

[10] Shelokar, P. S., Valadi K. Jayaraman, and Bhaskar D. Kulkarni. "An ant colony approach for clustering." Analytica Chimica Acta 509.2 (2004): 187-195.

[11] Korürek, Mehmet, and Ali Nizam. "A new arrhythmia clustering technique based on Ant Colony Optimization." Journal of Biomedical Informatics 41.6 (2008): 874-881.

[12] Storn R, Price K (2010) Differential evolution-a simple and efficient heuristic for global optimization over continuous spaces. J lobal Optim 23:689-694.

[13] Das, Swagatam, Ajith Abraham, and Amit Konar. "Automatic clustering using an improved differential evolution algorithm." IEEE Transactions on systems, man, and cybernetics-Part A: Systems and Humans 38.1 (2008): 218-237.

[14] Kwedlo, Wojciech. "A clustering method combining differential evolution with the K-means algorithm." Pattern Recognition Letters 32.12 (2011): 1613-1621.

[15] Kennedy J , Eberhart R . Particle swarm optimization. In: Proceedings of the 1995 IEEE international conference on neural networks; 1995. p. 1942-8.

[16] Ahmed, A., et al. "A review on particle swarm optimization algorithm and its variants to clustering highdimensional data." The Artificial Intelligence Review 44.1 (2015): 23.

[17] Alam, Shafiq, et al. "Research on particle swarm optimization based clustering: a systematic review of literature and techniques." Swarm and Evolutionary Computation 17 (2014): 1-13.

[18] Clerc M, Kennedy J (2000) The particle swarm-explosion, stability, and convergence in a multidimensional complex space. IEEE Trans Evol Comput 6(1):58-73

[19] Mendes R, Kennedy J, Neves J (2004) The fully informed particle swarm: simpler, maybe better. IEEE Trans Evol Comput 8:204-210

[20] Peram T, Veeramachaneni K, Mohan CK (2003) Fitnessdistance- ratio based particle swarm optimization. In: Proceedings of the IEEE swarm intelligence symposium, pp 174-181

[21] Naik A, Satapathy SC, Parvathi K (2013) A comparative analysis of results of data clustering with variants of particle swarm optimization. In: International conference on swarm, evolutionary, and Memetic computing, pp 180192

[22] Yohannes, M. S. "Solving economic load dispatch problem using particle swarm optimization technique." International Journal of Intelligent Systems and Applications 4.12 (2012):

[23] Ali Khazaee,"Heart Beat Classification Using Particle Swarm Optimization", International Journal of Intelligent Systems and Applications, vol.5, no.6, pp.25-33, 2013.

[24] Poonam Singhal, S. K. Agarwal, Narendra Kumar,"Advanced Adaptive Particle Swarm Optimization based SVC Controller for Power System Stability", International Journal of Intelligent Systems and Applications, vol.7, no.1, pp.101-110, 2015. 
[25] Rashedi E , Nezamabadi-Pour H , Saryazdi S . GSA: a gravitational search algo- rithm. Inf Sci 2009;179:2232-48.

[26] 20. Hatamlou, A., Abdullah, S., Nezamabadi-pour, H.: A Combined Approach for Clustering Based on K-means and Gravitational Search Algorithms. Swarm and Evolutionary Computation 6, 47-52 (2012)

[27] Hatamlou, A., Abdullah, S., Nezamabadi-pour, H.: Application of Gravitational Search Algorithm on Data Clustering. In: Yao, J., Ramanna, S., Wang, G., Suraj, Z. (eds.) RSKT 2011. LNCS, vol. 6954, pp. 337-346. Springer, Heidelberg (2011)

[28] Mirjalili, Seyedali, and Andrew Lewis. "The Whale Optimization Algorithm."Advances in Engineering Software 95 (2016): 51-67.

[29] Saidala, Ravi Kumar, and Nagaraju Devarakonda. "Improved Whale Optimization Algorithm Case Study: Clinical Data of Anaemic Pregnant Woman." Data Engineering and Intelligent Computing. Springer, Singapore, 2018. 271-281.

[30] Accepted: R. K. Saidala, N. Devarakonda. -A New Parallel Metaheuristic Optimization Algorithm and It‘s Application in CDM\|, Proceedings of the I2CT IEEE conference 2017, Pune.

[31] Ravi Kumar Saidala, Nagaraju Devarakonda "Improved Whale Optimization Algorithm using Clonal Selection Algorithm for finding optimal structures of data" Under review Information Sciences, Elsevier.

[32] Yevgeniy Bodyanskiy, Olena Vynokurova, Volodymyr Savvo, Tatiana Tverdokhlib, Pavlo Mulesa,"Hybrid Clustering-Classification Neural Network in the Medical Diagnostics of the Reactive Arthritis", International Journal of Intelligent Systems and Applications, Vol.8, No.8, pp.1-9, 2016.

\section{Authors' Profiles}

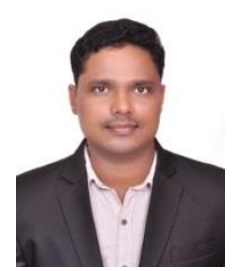

Ravi Kumar Saidala is a research scholar. $\mathrm{He}$ is pursuing his Ph.D. in Acharya Nagarjuna University (ANU). He completed his M. Tech in the year 2013 from Computer Science \& Engineering with the specialization of Digital Image Processing from ANU. He has worked as a guest faculty in the CSE department, ANU. He has received his B. Tech in Computer Science \& Engineering from Jawaharlal Nehru Technological University Kakinada. His research interests are Data Mining, Soft Computing, Digital Image Processing and Pattern Recognition.

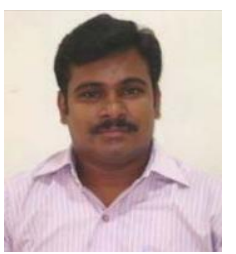

Dr. D. Naga Raju is the Professor and HOD of IT Department in Lakireddy Balireddy College of Engineering, Mylavaram. He was awarded his Ph.D in Computer Science \& Engineering from the Jawaharlal Nehru Technological University Hyderabad in the year 2014. He completed his master degree M. Tech (CSE) from Jawaharlal Nehru University (JNU), New Delhi in the year 2005. He completed his B. Tech (CSE) from Sri Venkateswara University, Tirupati in the year 2002. He has 16 years of teaching experience and published papers in various International journals, National and International conferences. His research interests are Data Mining, Soft Computing, Machine Learning and Pattern Recognition
How to cite this paper: Ravi Kumar Saidala, Nagaraju Devarakonda, "Multi-Swarm Whale Optimization Algorithm for Data Clustering Problems using Multiple Cooperative Strategies", International Journal of Intelligent Systems and Applications(IJISA), Vol.10, No.8, pp.36-53, 2018. DOI: 10.5815/ijisa.2018.08.04 